\title{
Problems Associated with Transferring of Engineering to Small Scale — Towards Theoretical Nanotechnology
}

\author{
J. KACZMAREK* \\ Institute of Fluid-Flow Machinery, Polish Academy of Sciences, J. Fiszera 14, 80-231 Gdańsk, Poland
}

(Received July 18, 2016)

\begin{abstract}
This paper is seen as a review of attempts carried out by author in order to indicate problems related to transferring of engineering to smaller scales having in mind theoretical aspects. Two main problems are indicated. The first one is associated with question what kind of theory is appropriate for realization of design of devices and processes related to smaller scales including nanoscale. The second problem is related to determination of source of precision which is necessary for realization of the design. Solution of both problems needs elaboration of appropriate theories. One indicates that unified mechanics of materials based on collection of dynamical systems with dimensional reduction is appropriate for future realization of the design process. This is multi-scale description which unifies discrete models related to atomic scale, including molecular dynamics, and more averaged continuum descriptions. In order to determine corresponding source of precision for small scale processes the vacuum medium mechanics is introduced. Within vacuum medium mechanics we are able to determine attractor, expressed on the most fundamental elementary particle level, and responsible for self-organization manifested in molecular processes. Vacuum medium mechanics is considered as fundamental theory. Therefore we should estimate to what degree such a theory is stable with respect to large set of experimental results. In particular one investigates consequences of vacuum medium mechanics for theoretical biology. One accentuates that mechanisms of biological evolution and source of precision for nanotechnology expressed by means of vacuum medium mechanics are interrelated. Theoretical biology and nanotechnology should be described in consistent way in order to cooperate in a future. Both theories: unified mechanics of materials and vacuum medium mechanics are seen as basis for further development of theoretical nanotechnology.
\end{abstract}

DOI: 10.12693/APhysPolA.130.1295

PACS/topics: 31.10.+z, 12.60.-i, 12.90.+b, 14.80.-j, 87.10.-e

\section{Introduction}

This paper is seen as a kind of review of attempts carried out for many years by the author and leading to indicating of problems placed on way towards transition of engineering to smaller scales. Appropriate determination of problems on this way has created starting point for elaboration of theory which should support corresponding engineering.

Contemporary engineering can be seen as considerable success for our civilization. This engineering predominantly related to macroscale has obtained systematic methodological and theoretical foundations. Engineering related to smaller scale is more accidental. Nevertheless it has also considerable success especially related to electronics. However activity in the smaller scale is not so systematic and supported by theory. Where from such a difference between various scale engineering appears?

First we should be aware where engineering comes from? Freedom of biological evolution is bounded by necessity of maintaining of integrity of biological organisms. New evolving forms and processes cannot destroy of evolving biological organisms. Consequently striving of biological organisms for attaining of new evolutionary possibilities of working in biological systems and environ-

*e-mail: jarekk@imp.gda.pl ment could sometimes destroy their integrity. In order to avoid this threat the evolving organisms could lead out these strivings beyond these organisms. This in turn has to be associated with loss of integrity of new structures and processes representing the new possibilities with corresponding organisms. We would say that this is just engineering. Consequently we come to the following remark:

Remark 1.1. Action of living organism leading to creation of new structures and processes characterized by loss of integrity with this organism and leading to attaining of new evolutionary possibilities of working in biological systems and environment is interpreted as engineering.

Loss of integrity increases number of degree of freedom for design and generation of new structures and processes since they are not bounded by mentioned previously integrity. Consequently we see that engineering and biological evolution are interrelated. Awareness of this fact can be especially important when we discuss engineering problems related to smaller scale. This is so since small scale engineering can be much more close to our organisms than large scale engineering.

\section{Properties of contemporary engineering}

In order to discuss problems of engineering related to smaller scale we should characterize properties of very successful engineering related to large scale. We would say that corresponding large scale advanced engineering 
has two important aspects: the first one is related to methods of design which depend on theory applied to this end, the second one is associated with source of precision which makes possible to realize in practice the design. Then whole process of making of construction should be associated to minimal degree with expensive experimental tests. In other words, advanced engineering is, to possible large degree, based on design with the aid of appropriate theory and corresponding numerical simulations, realization of the design with the aid of source of precision and a minimal set of experimental tests.

Let us note that theory which is systematically applied in engineering in reliable way is the elasticity theory. This theory allows us to express in numerical simulation strength of construction and dynamical properties in range where the constructions work. This theory is efficiently working in numerical simulations. Consequently we can obtain design of construction by numerical simulations. Next this construction can be precisely fabricated since precision of manufacturing of large scale elements is sufficiently good.

However, when we apply models of mechanics of materials to design of constructions then corresponding theories are not sufficiently reliable especially with respect to their range of validity. Let us note that application of theories of mechanics of materials transfer design of construction in fact to smaller scale which is frequently not manifested. We see that when the engineering touches smaller scale the situation is less stable.

The first immediate conclusion is that when we apply in systematic way models of mechanics of materials we need determination of their range of validity. Just range of validity of a model determined by some critical conditions makes possible transition to another model when the critical conditions are violated by processes in material. In such a case we are able to do more universal numerical simulations. Having this at our disposal we would be able to design properties of materials by means of numerical simulations. Creating of such a situation makes new challenges for mechanics of materials since today range of validity of models is rather not discussed. We come also to the conclusion that source of precision is less clearly determined in case of processing of materials and realization of corresponding designed material.

We see that contemporary engineering related to small scale is not so systematic as for larger scale. Nowadays we have to do with promotion of nanoscale engineering named nanotechnology. Let us discuss the two aspects of large scale engineering in relation to nanotechnology.

Nanotechnology is predominantly experimental engineering. It means that nanoscale devices are produced rather by experimental methods which are repeatable. Design of devices by numerical simulations is difficult since theory is not sufficiently elaborated. Furthermore, source of precision for nanotechnological processes is not determined in clear way.

The question is whether such a source of precission exist at all. We observe in living biological cells nanotech- nological processes which are realized with high precission. Let us mention transcription of mRNA, translation process leading to production of proteins, assembling of proteins into more complex structures having some functions. In the last case we see multiscale aspects of corresponding nanotechnology. All this suggests that a source of precission exists in considered processes and we need it also for nanotechnological processes.

We come to the conclusion that contemporary nanotechnology should tend to similar possibilities as we have in macroscopic engineering. At this moment however we have not at our disposal sufficiently good theory. Theory for nanotechnology is seen as set of theories related to small scale objects such as molecular dynamics, quantum mechanics or quantum chemistry which seem to be not sufficiently good for current needs.

Source of precision for nanotechnology should be related to larger degree to dynamics of processes. This point of view is justified by fact that role of dynamics increases when scale decreases. Therefore we come to the conclusion that source of precision should be associated with a self-organization process related to sufficiently small scale. This is so since self-organization is closely related just to dynamics of processes.

We would say that contemporary nanotechnology avoids the question how to describe source of precision. Evidently, such a description cannot be obtained from quantum theories which are linear in their initial concepts. They are sometimes modified to nonlinear theories. However, corresponding nonlinearities do not lead to description of self-organization processes seen as responsible for precision of nanotechnological processes. We should have at our disposal a theory which indicates an attractor on the most fundamental elementary particle level responsible for self-organization which is manifested especially in molecular processes.

Above discussion accentuates role of theory in small scale engineering. It justifies directly development of theoretical nanotechnology which would be entirely devoted to design and manufacturing of nanotechnological devices within numerical simulations. Then, theoretical nanotechnology would support real nanotechnology by appropriate understanding of processes by theory and indicating of source of precision for their practical realization.

Let us note that the term theoretical nanotechnology is applied today. Let us mention Handbook of Theoretical and Computational Nanotechnology [1] for instance. However, it seems that this term is applied to all theories which are able to support nanotechnology. Let us mention molecular dynamics, quantum mechanics, or quantum chemistry. However, mentioned theories are not elaborated for nanotechnology. All they are generated in various stages of development of theoretical physics and next adopted by nanotechnology. As a result of this important problems such as determination of source of precision for nanotechnological processes or elaboration of consistent and universal theory for design of nanotech- 
nological processes and devices are not considered. The term theoretical nanotechnology is considered in this paper as theory developed with intention to fulfill requirements defined directly by nanotechnology. It is expected also that such a theory should be consistent in description of all processes involved in design and manufacturing of corresponding devices.

\section{Concept of theory directed to design of structures and processes related to small scales \\ 3.1. Requirements for unified theory of processes in materials}

We should characterize general requirements which should be satisfied by theory appropriate for application to design process considered within engineering related to smaller scales.

We expect that theoretical description should take into account processes related to atomic scale since this scale is close to nanotechnological processes. On the other hand, process of design frequently takes into account properties which follow from various scales. Consequently, we should have at our disposal description of all processes within one theoretical framework including larger scales processes predominantly expressed by continuum descriptions. Above discussion suggests that we should consider multiscale description within mechanics of materials as theory appropriate for small scale engineering.

Theoretical description should have large range of validity. It is especially important when we consider processes which are characterized by a qualitative change. Let us mention in this place phase transformation for instance. Then, descriptions of processes before phase transformation and after the phase transformation frequently are qualitatively different. Consequently, we should have at our disposal methods of efficient transition from one description to another. This is important for efficient numerical simulations for more complex processes.

Above property means that for each description with a given quality we should have at our disposal corresponding range of its validity. In other words, when we have some equations within a description then we should have also some conditions which determine range of validity of corresponding equations. Then, violation of mentioned conditions determines necessity to transition to another description. Creation of such a situation in theoretical description is usually not considered as a custom.

Summarizing, above requirements suggest need of creating of unified mechanics of materials in order to make possible numerical simulations in whole range where processes are continued. Only such a theory allows us to design devices associated with all arbitrary processes in material.

It is assumed that general framework for unified mechanics of materials should be based on theory called here collection of dynamical systems with dimensional reduction. Therefore this theory will be discussed in what follows.

\subsection{General assumptions related to collection of dynamical systems}

In accordance with previous discussion we see that diminishing of scale leads to increasing role of dynamics of processes. Consequently we should apply dynamical system approach when we would like to describe the most elementary processes in small scale. Dynamical systems theory is an important branch of mathematics [2] directed towards modelling of evolution of systems and is widely applied in physics and mechanics. See for instance [3]. Our considerations on collection of dynamical systems are based on $[4,5]$.

We expect considerable complexity of phenomena related to small scale. Not all properties of these processes are interesting for us. Thereby, we would like to have at our disposal some methods of simplification of this complex physical picture in order to refine more interesting for us properties of the system modelled. A possible way for such a simplification is to distinguish some dynamical subsystems. Then, obtained structure of dynamical subsystems would be a base for the dimensional reduction procedure.

We introduce first a dynamical system aimed at description of phenomena on the most elementary level. Such a system is called here the elementary dynamical system $(E D S)$ and is given in a general form

$$
\dot{\boldsymbol{\varphi}}=L(\boldsymbol{\varphi}, \boldsymbol{f}) \text {, }
$$

where $\varphi \in \mathcal{M}_{\varphi}$ is the variable of this system, $\mathcal{M}_{\varphi}$ is a space of admissible values of this variable, $\boldsymbol{f} \in \mathcal{F}$ represents external interactions acting on this system and $\mathcal{F}$ stands for space of admissible values of $\boldsymbol{f}$.

External interactions are not always expressed in a simple form given by $\boldsymbol{f}$. Sometimes, they appear as interactions with other dynamical systems. Therefore, we introduce also an extended dynamical system

$$
\dot{\varphi}^{r}=L\left(\boldsymbol{\varphi}^{r}, \boldsymbol{f}^{r}\right),
$$

where $\varphi^{r}=\left\{\boldsymbol{\varphi}, \boldsymbol{\varphi}^{e}\right\} \in \mathcal{M}_{\varphi r}$ and $\boldsymbol{f}^{r}=\left\{\boldsymbol{f}, \boldsymbol{f}^{e}\right\} \in \mathcal{F}_{R}$. In other words the dynamical system (1) is a part of that one defined by (2) and can be viewed as a particular case of (2). As a result of this an external dynamical system with variable $\varphi^{e}$ can be additionally distinguished, as a model of external interactions acting on EDS. This gives a possibility of discussing larger class of interactions of (1) with an external world.

The dynamical system (1) describes more elementary processes and its form is, by assumption, the most complex. We tend towards simplifications of this system. To this end we introduce a partition of (1) onto a collection of $P$ dynamical systems. This is carried out by partition of variable $\boldsymbol{\varphi}=\left\{\boldsymbol{\varphi}_{h}\right\}, h \in I_{P}=\{1,2, \ldots, P\}$.

In order to use this partition for further simplifications we introduce also additional notations, sets and mappings. Let $\prod_{h} \mathcal{M}_{h}$ be Cartesian product of sets $\mathcal{M}_{h}$, where $\mathcal{M}_{h}$ stands for set of admissible values of $\boldsymbol{\varphi}_{h}$. Let us introduce $\mathcal{M}_{\Pi} \subset \prod_{h} \mathcal{M}_{h}$ as a subset of the Cartesian product. Then, $\mathcal{M}_{\Pi}$ consists of $\boldsymbol{\varphi}=\left\{\boldsymbol{\varphi}_{h}\right\}$ which are possible solutions of Eq. (1). We consider also a projection $\pi_{h}: \mathcal{M}_{\Pi} \rightarrow \mathcal{M}_{h}$ in the Cartesian product. 
Let us distinguish also a collection of dynamical systems for the extended system and a set of indexes $I_{R}$ related to them. Then, $I_{R}=I_{P} \cup I_{E}, I_{P} \cap I_{E}=\emptyset$, where $I_{P}$ is related to $(1)$ and $I_{E}=I_{R}-I_{P}$ is connected with the external system. Furthermore we distinguish a group of dynamical subsystems $I_{G} \subset I_{P}$ by selection of corresponding indexes. Then, $I_{O}=I_{R}-I_{G}$ represents indexes defining external with respect to $I_{G}$ dynamical subsystems within (2).

New simplified equations have to be based on balance of mass and energy equations as the most fundamental physical laws for mechanics of materials. In order to create such equations for the collection of dynamical systems we should have at our disposal a set of additional notions making possible to formulate them. Therefore we introduce the following assumptions which represent general properties of the collection of dynamical systems distinguished within the elementary dynamical system $[4,5]$ : 1. Subsystems are distinguished by determination of groups of variables $\varphi_{h}=\left\{\varphi_{h \alpha}\right\}$, where $\alpha \in I_{A h}, I_{A h}$ is a finite set related to separate $h$-th subsystem and $h \in I_{P}$.

2. There exists a function $\bar{m}_{h}\left(\boldsymbol{\varphi}_{h}\right)=\left\{m_{h 1}, \ldots, m_{h \beta_{h}}\right\}$ which assigns a set of masses for $h$-th subsystem. The total mass of the system is $m_{h}=\sum_{i} m_{h i}$. We have also that $\sum_{h} \beta_{h}=N$, where $N$ is the total number of masses. Then, the functions $\tilde{m}: \mathcal{M}_{\Pi} \rightarrow R^{P}$ with property $\pi_{h} \circ \tilde{m}\left(\left\{\boldsymbol{\varphi}_{h}\right\}\right)=m_{h}$ and $m: \mathcal{M}_{\Pi} \rightarrow R$, $m\left(\left\{\boldsymbol{\varphi}_{h}\right\}\right)=\sum m_{h}$ determine the total mass related to each subsystem and the total mass related to (1), respectively.

3. There exists a function $\tilde{E}: \mathcal{M}_{\Pi} \rightarrow R^{P}, \pi_{h}$ 。 $\tilde{E}\left(\left\{\varphi_{h}\right\}\right)=E_{h}$ which assigns a value of energy to each of subsystems and $E: \mathcal{M}_{\Pi} \rightarrow R, E\left(\left\{\varphi_{h}\right\}\right)=\sum_{h} E_{h}$ determines the total energy related to (1).

4. There exists a family of mappings $J_{i j}: \mathcal{M}_{\Pi} \rightarrow R$, $i, j \in I_{P}, J_{i j}\left(\left\{\boldsymbol{\varphi}_{h}\right\}\right)=J_{i j}$ called flux of mass from $j$-th subsystem to $i$-th subsystem and $J_{i j}+J_{j i}=0, J_{i i}=0$.

5. There exists a family of mappings $W_{i j}: \mathcal{M}_{\Pi} \rightarrow R$, $i, j \in I_{P}, W_{i j}\left(\left\{\boldsymbol{\varphi}_{h}\right\}\right)=W_{i j}$ called flux of energy from $j$-th subsystem to $i$-th subsystem and $W_{i j}+W_{j i}=0$, $W_{i i}=0$.

6. Source of mass is determined by the function $c$ : $\mathcal{M}_{\Pi} \rightarrow R^{P}, c\left(\left\{\boldsymbol{\varphi}_{h}\right\}\right)=\left\{c_{i}\right\}$. Then, $c_{i}=\pi_{i} \circ c\left(\left\{\boldsymbol{\varphi}_{h}\right\}\right)$ can be considered for each subsystem of the whole system and stands for source of mass in $i$-th subsystem.

7. Source of energy is determined by the function $R$ : $\mathcal{M}_{\Pi} \rightarrow R^{P}, R\left(\left\{\boldsymbol{\varphi}_{h}\right\}\right)=\left\{R_{i}\right\}$. Then, $R_{i}=\pi_{i} \circ R\left(\left\{\boldsymbol{\varphi}_{h}\right\}\right)$ can be considered for each subsystem of the whole system and stands for source of energy in $i$-th subsystem.

8. Geometrical objects can be assigned to each subsystem. This is carried out with the help of mappings $G_{x}: \mathcal{M}_{\Pi} \rightarrow E_{e}^{P}, G_{L}: \mathcal{M}_{\Pi} \rightarrow\left(2^{E_{e}}\right)^{P}, G_{S}: \mathcal{M}_{\Pi} \rightarrow$ $\left(2^{E_{e}}\right)^{P}, G_{V}: \mathcal{M}_{\Pi} \rightarrow\left(2^{E_{e}}\right)^{P}$, where $E_{e}$ is the Euclidean space. The map $G_{x}$ assigns some distinguished points to subsystems, $G_{L}$ introduces one-dimensional, $G_{S}$ twodimensional, $G_{V}$ three-dimensional geometrical objects considered as subsets of $E_{e}$ and accompanied by distin- guished subsystems.

All discussed assumptions and functions can also be introduced for the extended dynamical system (2). They enable us considerations of balance of mass and energy equations for collection of dynamical systems. Geometrical elements allow us to create various relations associated with geometry of our space in relation to dynamical subsystems.

\subsection{Balance of mass and energy for collection of dynamical systems}

We tend towards expression of dimensionally reduced equations for collection of dynamical systems. General laws expressed by balance of mass and energy equations are seen as fundamental for this description. In particular continuum mechanics equations considered as more averaged description in comparison with atomic scale models for instance have to be based on such equations.

Assumptions introduced above for collection of dynamical systems make possible analysis of interchange of mass between subsystems as well as to consider possible sources of mass which appear within subsystems. Then, we are able also to express the balance of mass equation for collection of dynamical systems in the following form:

$$
\begin{aligned}
& \sum_{i \in I_{G}}\left(\dot{m}_{i}-c_{i}\right)+\sum_{i, j \in I_{G}} J_{i j}+\sum_{i \in I_{G}, j \in I_{O}}\left(J_{i j}+J_{j i}\right) \\
& +\sum_{i, j \in I_{O}} J_{i j}+\sum_{i \in I_{O}}\left(\dot{m}_{i}-c_{i}\right)=0 .
\end{aligned}
$$

Sum of masses interchanged between subsystems within $I_{G}$ without any interchange with an external subsystems is equal to zero. Thus, we have $\sum_{i, j \in I_{G}} J_{i j}=0$. As a result we can express the balance of mass equation connected with group of subsystems represented by $I_{G}$ with the help of formula

$$
\sum_{i \in I_{G}}\left(\dot{m}_{i}-c_{i}+\sum_{j \in I_{O}} J_{i j}\right)=0 .
$$

In Eq. (4) the terms $J_{i j}$ describing interchange of mass with external system $I_{O}$ appear. Then, Eq. (4) is not entirely determined. Therefore we should introduce an additional condition

$$
J_{i j}=\bar{J}_{i j}, j \in I_{O} \text {. }
$$

Form of $\bar{J}_{i j}$ should be postulated by a kind of constitutive equations.

The balance of energy equation has similar structure as the balance of mass equation and is given by

$$
\begin{aligned}
& \sum_{i \in I_{G}}\left(\dot{E}_{i}-R_{i}\right)+\sum_{i \in I_{G}, j \in I_{O}}\left(W_{i j}+W_{j i}\right) \\
& \quad+\sum_{i \in I_{O}}\left(\dot{E}_{i}-R_{i}\right)=0 .
\end{aligned}
$$

The balance of energy equation for group of subsystems $I_{G}$ interacting with groups of subsystems $I_{O}$, is given by

$$
\sum_{i \in I_{G}}\left(\dot{E}_{i}-R_{i}+\sum_{j \in I_{O}} W_{i j}\right)=0
$$




$$
W_{i j}=\bar{W}_{i j}, j \in I_{O} .
$$

Let us note that $E_{i}$ and $W_{i j}$ depend, in general, on state of the whole system in accordance with assumptions 3 and 5 .

Equations (4), (5) and (7), (8) represent a general form of balance of mass and energy equations related to arbitrary distinguished group of subsystems within the collection of dynamical systems.

\subsection{Description of the dimensional reduction procedure}

We try to define the dimensional reduction procedure as a way of determination of simplified equation in comparison with an elementary dynamical system. To this end we have to determine variables which are applied in new equations. Furtheremore, various interactions between subsystems should be taken into account. Mathematical representation of corresponding interactions should be considered with the aid of quantities introduced to this end. They can be similar to gradients or higher gradients of variables introduced. However, we do not define gradients with respect to space variables here since our subsystems are finite in some sense or have finite size when we apply geometrical objects assigned to subsystems. Consequently we should discuss how corresponding quantities should be introduced. Let us note that the name "dimensional reduction procedure" means that obtained equations will be finite-dimensional with smaller number of degree of freedom than the elementary dynamical system. The dimensional reduction procedure is a method of transition from elementary dynamical system to a simpler dynamical system having less number of degree of freedom. The first step of the dimensional reduction consists in option of new variables. Let $\boldsymbol{d}=\left\{\boldsymbol{d}_{h}\right\}, h \in I_{R}$ be a set of new variables which allows to describe, approximately, physical states represented by miscellaneous $\varphi^{r}$. Let $\overline{\mathcal{M}}$ stands for space of admissible values of $\boldsymbol{d}$. $\boldsymbol{d}_{h}$ describes behaviour of $h$-th subsystem in a simplified form. We have by assumption that $\operatorname{dim} \overline{\mathcal{M}}$ is considerably smaller than $\operatorname{dim} \mathcal{M}_{\varphi}$.

Let $V_{T}=\{\boldsymbol{\varphi}(t): t \in T\}, V_{T r}=\left\{\boldsymbol{\varphi}^{r}(t): t \in T\right\}$ and $\bar{V}_{T}=\{\boldsymbol{d}(t): t \in T\}$. The first element of the dimensional reduction procedure is based on introduction of a map$\operatorname{ping} \pi_{T}: V_{T r} \rightarrow \bar{V}_{T}$ which assigns dimensionally reduced process $\boldsymbol{d}(t)$ to $\varphi^{r}(t)$ on the time interval $T$.

We introduce also $\mathcal{F}_{T}=\{\boldsymbol{f}(t), t \in T\}$ and $\overline{\mathcal{F}}_{T}=$ $\{\overline{\boldsymbol{f}}(t), t \in T\}$ with mapping $\pi_{f T}: \mathcal{F}_{T} \rightarrow \overline{\mathcal{F}}_{T}$ which transforms forces between elementary and reduced system.

We can introduce a division of variables $\boldsymbol{d}(t)$ into parts $\boldsymbol{d}^{p}(t)$ and $\boldsymbol{d}^{e}(t)$ as corresponding with $E D S$ and external dynamical system in smaller scale. Similar partition is possible for $\boldsymbol{f}(t)$ and $\overline{\boldsymbol{f}}(t)$ since they are considered here for extended system. Thus, we carry out the dimensional reduction for the extended dynamical system (2). However, our interest is related mainly to $E D S$ given by (1).

The external dynamical system is purposed to model a larger class of external interactions. Thereby, the external interactions related to $E D S$ are given by $\boldsymbol{f}(t)$ and $\boldsymbol{f}^{r}$ and furthermore by form of the external system. In particular external dynamical system can be reduced to the most simple form represented by static forces. Thus, the dimensional reduction procedure should represent different procedures for $E D S$ and for the external system in general. This takes place since discussed systems have separate status in the framework of the presented description. However, we will use $\varphi$ instead of $\varphi^{r}$ and $f$ instead of $\boldsymbol{f}^{r}$ in what follows if it does not lead to mistake.

Let us introduce an operator $\mathcal{L}: \mathcal{M}_{\varphi r} \rightarrow \mathcal{F}_{r}$ constructed with the help of Eq. (2) as $\mathcal{L}(\varphi)=\tilde{\mathcal{L}}(\boldsymbol{\varphi}, \dot{\varphi})$, where $\tilde{\mathcal{L}}$ is obtained from equivalent to Eq. (2) in the form $\tilde{\mathcal{L}}(\boldsymbol{\varphi}, \dot{\varphi})=\boldsymbol{f}^{r}$. Then, the operator acting on processes $\mathcal{L}_{T}: V_{T r} \rightarrow \overline{\mathcal{F}}_{T}$ is induced directly by means of $\mathcal{L}$ for each $t \in T$. Let us consider a diagram

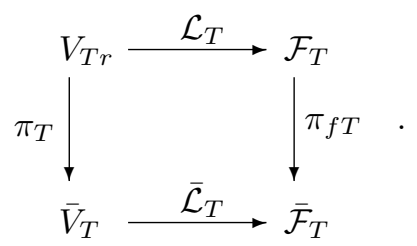

Accordingly, the initially introduced equation $\mathcal{L}_{T}(\boldsymbol{\varphi}(t))=\boldsymbol{f}(t)$ induces, owing to assumed $\pi_{T}$ and $\pi_{f T}$, a dimensionally reduced equation

$$
\overline{\mathcal{L}}_{T}(\boldsymbol{d}(t))=\overline{\boldsymbol{f}},
$$

where $\overline{\mathcal{L}}_{T}=\pi_{f T} \circ \mathcal{L}_{T} \circ \pi_{T}^{-1}$. The operator $\overline{\mathcal{L}}_{T}$ can be determined with the help of solutions of Eq. (2) and postulated mappings $\pi_{T}, \pi_{f T}$ for each value of $\boldsymbol{d}(t)$. However, structure of the operator $\overline{\mathcal{L}}_{T}$ is not discussed yet since too small number of assumptions is done at this moment.

Let us discuss a similar diagram for a group of subsystems $I_{G} \subset I_{P}$. Then, we carry out the following decomposition: $V_{T r}=V_{T G} \times V_{T O}=\left\{\boldsymbol{\varphi}(t)=\left\{\boldsymbol{\varphi}_{g}(t), \boldsymbol{\varphi}_{l}(t)\right\}, g \in\right.$ $\left.I_{G}, l \in I_{O}\right\}$. The symbol " $\times$ " does not stand for Cartesian product operation but means a kind of relation in which $\varphi_{g}, \varphi_{l}$ together create a solution of (2). We introduce also decomposition $\mathcal{F}_{T}=\mathcal{F}_{T G} \times \mathcal{F}_{T O}$ in similar way.

Owing to above decompositions of domain and range, the operator $\mathcal{L}_{T}$ can be expressed as $\mathcal{L}_{T}=$ $\mathcal{L}_{T\left(\varphi_{l}\right)}\left(\left\{\boldsymbol{\varphi}_{g}(t)\right\}\right) \times \mathcal{L}_{T\left(\varphi_{g}\right)}\left(\left\{\boldsymbol{\varphi}_{l}(t)\right\}\right)$.

The operator $\mathcal{L}_{T\left(\varphi_{l}\right)}: V_{T G} \rightarrow \mathcal{F}_{T G}$ depends on $\varphi_{l}$. Usually, not all $\varphi_{l} \in V_{T O}$ are necessary for determination of $\mathcal{L}_{T\left(\varphi_{l}\right)}$.

Let $I_{G O}$ stands for a set of indexes which indicate variables necessary for determination of the operator $\mathcal{L}_{T G}:=\mathcal{L}_{T\left(\varphi_{l}\right)}$. Then, the operator $\mathcal{L}_{T}$ can be expressed in simplified form as $\mathcal{L}_{T G} \times T_{C}$, where $T_{C}=T_{C}\left(\boldsymbol{\varphi}_{g}, \boldsymbol{\varphi}_{c}\right)$, $c \in I_{G O}$. The operator $T_{C}$ can have considerably simpler form for determination of external interactions in comparison with $\mathcal{L}_{T}$. With the help of $T_{C}$ we are able to introduce additional equations necessary for determination of $\boldsymbol{\varphi}_{c}$ defining form of $\mathcal{L}_{T G}$ within $\mathcal{L}_{T}$. The space $V_{T r}$ is reduced to $V_{T G} \times V_{T C}$ in this case.

The modified diagram (9) can be expressed now in the following form: 


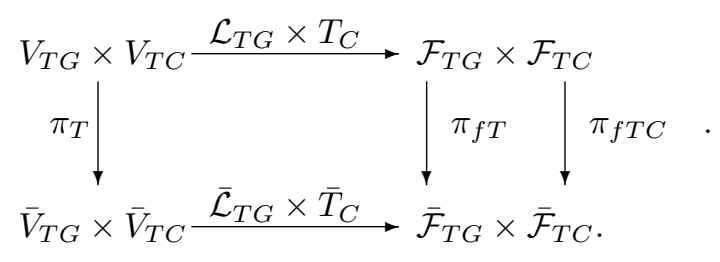

Accordingly, the dimensionally reduced equation describing evolution of group of subsystems is given by

$$
\begin{aligned}
& \overline{\mathcal{L}}_{T G\left(\boldsymbol{d}_{c}\right)}\left(\left\{\boldsymbol{d}_{g}(t)\right\}\right)=\left\{\overline{\boldsymbol{f}}_{g}\right\}, \\
& \bar{T}_{C c}\left(\boldsymbol{d}_{g}(t), \boldsymbol{d}_{c}(t)\right)=\mathcal{B}_{c} .
\end{aligned}
$$

We see that separate status of dimensional reduction related directly to $E D S$ and that one related to external dynamical system applied in description of interactions leads to introduction by (13) of various forms of conditions similar to boundary conditions in continuum description.

Diagram (11) allows to solve Eqs. (12), (13) by means of solutions of Eq. (2) and postulated form of $\pi_{T}, \pi_{f T}$, $\pi_{f T C}$. However, we would like to have a precise structure of $\overline{\mathcal{L}}_{T G}$ and $\bar{T}_{C}$ in order to have at our disposal possible to solution, dimensionally reduced equations without using (2) incessantly.

We suggest to obtain corresponding equations by means of postulating of the skeletal dynamical system $S D S(\boldsymbol{C})$ which depends on family of constants $\boldsymbol{C}$. The role of $S D S$ is to represent a larger class of systems which encompass approximately Eqs. (12), (13). Accordingly, the general form of $S D S$ in case of (12), (13) can be expressed as $\left\{\overline{\mathcal{L}}_{T G}, \bar{T}_{C}\right\}(\boldsymbol{C})\left(\boldsymbol{d}_{g}, \boldsymbol{d}_{c}\right)=\{\overline{\boldsymbol{f}}, \mathcal{B}\}$. It means that postulated $S D S$ was derived taking into account general premises applied in Eqs. (12) and (13). Then, with the help of an identification method, we can determine $\boldsymbol{C}=\overline{\boldsymbol{C}}$ and as a result of this we obtain also a reduced dynamical system $R D S=S D S(\overline{\boldsymbol{C}})$. Finally, $R D S$ represents an approximation of Eqs. (12) and (13).

General structure of $S D S$ should take into account fundamental laws in the first stage of formulation. In case of mechanics of materials such laws are expressed by balance of energy and balance of mass equations. They have been previously formulated for collection of dynamical systems.

Kind of variables $\boldsymbol{d}=\left\{\boldsymbol{d}_{h}\right\}$ also decides on form of $S D S$. Furthermore, interactions between subsystems should be expressed by variables. To this end we introduce a function of kinematic dependence between subsystems.

Let $H_{d h}=\left\{\boldsymbol{d}_{j}: j \in I_{h}^{a}\right\}$ be a set of values of the variable $\boldsymbol{d}_{j}$ determined on a set of subsystems indexed by elements of a set $I_{h}^{a}$. $I_{h}^{a}$ represents all subsystems which interact with the $h$-th one.

Let us introduce a function $a_{h}: H_{d h} \rightarrow V_{a h}$, where $V_{a h}$ is a linear space. Accordingly, the function $a_{h}$ assigns an element of the linear space connected with $h$-th subsystem to a set of values of variables $\boldsymbol{d}_{j}$ related to interacting subsystems. The function $a_{h}$ is called the function of kinematic dependence between subsystems. Form of this dependence is embodied in elements of $V_{a h}$. The function $a_{h}$ has structure which allow us to introduce dependence of quantities on gradients and also higher gradients of introduced variables in our discrete systems necessary for description of various interactions between subsystems.

We introduce also an additional concept of taking into account interactions to dimensionally reduced system. In order to describe interactions between different systems, a value of $g_{h}$ type in a given point $\boldsymbol{X}$ assigned to a subsystem can be useful. Consequently, we admit possibility of introducing this kind of quantity. We assume that it is possible to introduce a transmission function $\mathcal{T}_{x}\left(\left\{g_{h}\right\}\right)=g_{x}$ which assigns a value of $g_{x}$ in the point $\boldsymbol{X}$ to the set of values $\left\{g_{h}\right\}$. We introduce $g_{x}$ as dependent on $\left\{g_{h}\right\}$. Consequently, no additional degrees of freedom is produced by this kind of function. This method allow us to describe interactions in context of a geometry assigned to subsystems.

The transmission function can be introduced also more generally without inerpretation of variables in context of space position. Then, $\mathcal{T}_{\alpha}\left(\left\{g_{h}\right\}\right)=g_{\alpha}$ assigns additional variables to the set of existing variables. Sometimes such transformation allows to model interactions in more convenient way.

Application of this function is possible with the help of the function $a_{h}$ which depends on set of values of fields distributed in space and describes just interactions. In particular, the function $a_{h}$ can also be defined with the help of $g_{x}$ or $g_{\alpha}$.

Masses related to dynamical system also undergo dimensional reduction. This is introduced by $\pi_{M}\left(\left\{m_{h i}\right\}\right)=$ $\left\{M_{h p}\right\}$. Thus, $M_{h p}$ are inertia coefficients related to $h$-th subsystem and are present in the form of $S D S$.

Summing up these considerations we have obtained some general premises for formulating the skeletal dynamical system. First, the balance of energy and mass equations should be taken into account. Next, quantities which appear in these equations should be plotted with $\boldsymbol{d}_{h}$ and $a_{h}(\boldsymbol{d})$ as well as with new inertia characteristics, in order to express state and interactions of subsystems. The transmission functions can be helpful to this end.

Let us discuss a concept of an identification method of constants defining $R D S$ from $S D S(\boldsymbol{C})$. Let $C=\{\psi(t)$ : $\psi \in \overline{\mathcal{M}}, t \in T\}$ be a space of continuous time processes in $\overline{\mathcal{M}}$ with a metric $\rho: C \times C \rightarrow R_{+} \cup\{0\}$. We can construct two kinds of processes. The first one is based on solution $\boldsymbol{\varphi}\left(\boldsymbol{\varphi}_{0}, \boldsymbol{f}\right)(t)$ of Eq. (2) and have the form $\pi_{T}\left(\boldsymbol{\varphi}\left(\boldsymbol{\varphi}_{0}, \boldsymbol{f}\right)(t)\right)$. The second one is created by the skeletal dynamical system with assumed constants $\boldsymbol{C}$. Thus, we have a solution of equations of $S D S$ as $\left.\boldsymbol{d}\left(\boldsymbol{C}, \pi\left(\boldsymbol{\varphi}_{0}\right), \overline{\boldsymbol{f}}\right)\right)(t)$, where $\overline{\boldsymbol{f}}(t)=\pi_{f T}(\boldsymbol{f}(t))$. Let us consider the function

$$
\begin{aligned}
& h\left(\boldsymbol{\varphi}_{0}, \boldsymbol{f}\right)= \\
& \quad \inf _{\boldsymbol{C} \in \mathcal{C}_{e}} \rho\left(\boldsymbol{d}\left(\boldsymbol{C}, \pi\left(\boldsymbol{\varphi}_{0}\right), \overline{\boldsymbol{f}}\right)(t), \pi_{T}\left(\boldsymbol{\varphi}\left(\boldsymbol{\varphi}_{0}, \boldsymbol{f}\right)(t)\right)\right),
\end{aligned}
$$

where $\mathcal{C}_{e}$ is an admissible set of constants for which energy of the system is well approximated. It means that for each $\boldsymbol{C} \in \mathcal{C}_{e}, E(\boldsymbol{\varphi})(t) \approx E(\boldsymbol{d})(t)$. 
Let $C^{*}$ stands for constants for which the function $h$ attains a minimum. Then, a satisfactory approximation should have the property that $\boldsymbol{C}^{*}$ exhibits a weak dependence on $\boldsymbol{d}_{0}$ and $\boldsymbol{f}$. This, in turn, is connected with assumed functions $\pi_{T}$ and $S D S$ which reflect correctness of averaged modelling. Finally, we have to choose a constant $\overline{\boldsymbol{C}}$ from the set of $\boldsymbol{C}^{*}$ by an averaging method. Then

$$
\left.\overline{\boldsymbol{C}}=\operatorname{Av}\left\{\boldsymbol{C}^{*}: \boldsymbol{C}^{*}\left(\boldsymbol{d}_{0}, \overline{\boldsymbol{f}}\right), \boldsymbol{d}_{0} \in \overline{\mathcal{M}}, \overline{\boldsymbol{f}} \in \overline{\mathcal{F}}\right)\right\},
$$

where Av means the averaging operation. Obtained constants $\bar{C}$ determine a dimensionally reduced dynamical system $R D S(\overline{\boldsymbol{C}})$. By means of formulae (14), (15) an approximation and identification procedure denoted generally by app is established.

We can discuss also another kind of an approximation procedure. Let us assume that there is a relation $\mathcal{R}_{a p p}$ between variables and forces of $S D S$ and constants $\boldsymbol{c}_{E D S}$ considered in the elementary dynamical system.

Let us consider a system of equations

$$
\begin{aligned}
& \left\{\overline{\mathcal{L}}_{T G}, \bar{T}_{C}\right\}\left(\boldsymbol{C}, \boldsymbol{d}_{g}, \boldsymbol{d}_{c}\right)=\{\overline{\boldsymbol{f}}, \mathcal{B}\}, \\
& \mathcal{R}_{\text {app }}\left(\boldsymbol{d}_{g}, \boldsymbol{d}_{c}, \overline{\boldsymbol{f}}, \mathcal{B}_{c}, \boldsymbol{c}_{E D S}\right)=0 .
\end{aligned}
$$

We assume that Eq. (16) and the relation $\mathcal{R}_{a p p}$ create together such a system of equations which enables to determine $\overline{\boldsymbol{C}}=\boldsymbol{C}\left(\boldsymbol{c}_{E D S}\right)$. In such a case the reduced dynamical system is also determined.

Summing up these considerations let us notice that the following general procedure is established: $\{E D S, D R\} \rightarrow R D S(\overline{\boldsymbol{C}})$. It means that the dimensional reduction procedure $D R=\left\{\pi_{T}, \pi_{f T}, S D S, a p p\right\}$ acting on an elementary dynamical system (2) leads to obtaining the reduced dynamical system $R D S$. Consequently, $R D S$ is considered as describing approximately, evolution of our initially introduced physical system.

\subsection{Continuum with finite-dimensional fields}

Continuum mechanics is important method of modelling widely applied also in mechanics of materials. Therefore we should discuss how we see place of continuum mechanics on our way leading to unified mechanics of materials. Formal mathematical foundations of continuum mechanics are done within rational mechanics [6]. We introduce here modification of continuum mechanics with special regard to placing it in the more general theory of collection of dynamical systems with dimensional reduction.

Continuum mechanics describes properties of a material in more averaged way than this is done by molecular dynamics for instance. However, fields on continuum create predominantly infinite-dimensional spaces. On the other hand, molecular dynamics equations have finite number of degree of freedom. Thereby, equations of continuum mechanics should be reformulated in order to have smaller number degree of freedom than the atomistic models have. Only then the continuum theory can be considered as simpler and can be obtained by means of the dimensional reduction procedure.

The idea of introducing finite dimensional fields consists in assumption on validity of balance equations not for all subbodies of the body $\mathcal{B}$ but only for their determined family $\mathcal{K}$. It is in fact a generalization of classical formulation since all subbodies create a particular case of $\mathcal{K}$.

The notions of the classical continuum should be modified with respect to a finite family of subbodies $\mathcal{K}$ in order to obtain spaces of finite dimensional fields. We would like also to connect notions related to continuum with an elementary dynamical system. To this end mappings defining geometrical objects related to dynamical subsystems will be used. They have been introduced in general assumptions characterizing collection of dynamical systems.

Consequently, we can consider mappings $G_{x}, G_{L}, G_{S}$, $G_{V}$ which assign zero-, one-, two- and three-dimensional geometrical objects to each subsystem correspondingly.

Let us apply the special case of the map $G_{V}: \mathcal{M}_{\Pi} \rightarrow$ $\left(2^{E_{e}}\right)^{P}$ as $G_{V}=G_{K}$. Let $\mathcal{M}_{K}=\{\mathcal{K}\}$ be a set of possible families of $\mathcal{K}=\left\{K_{h}\right\}$, where $K_{h} \subset E_{e}$ and $\operatorname{int} K_{g} \cap \operatorname{int} K_{h}=\emptyset, g, h \in I_{P}$. Then, $G_{K}: \mathcal{M}_{\Pi} \rightarrow$ $\mathcal{M}_{K}, G_{K}\left(\left\{\varphi_{h}\right\}\right)=\mathcal{K}$. We introduce also the mapping $G_{K h}\left(\left\{\varphi_{h}\right\}\right)=K_{h}$.

Consequently, the mapping $G_{K}$ introduces threedimensional disjoint subsets of $E_{e}$ which will be further interpreted as partition of the body $\mathcal{B}$ into the set of subbodies $K_{h}$ and $\bigcup K_{h}$.

Let us note that $G_{K}\left(\left\{\varphi_{h}\right\}(t)\right)=\left\{K_{h}\right\}(t)$ describes evolution of $\mathcal{B}(t)=\bigcup_{h} K_{h}(t)$ in time. $K_{h}(t)$ will be also denoted by $\chi_{t}\left(K_{h}\right)$ in what follows.

Definition 3.1: The body associated with the dynamical system $\dot{\varphi}=L(\boldsymbol{\varphi}, \boldsymbol{f})$ is defined with the help of mapping $G_{K}$ as $\mathcal{B}_{\varphi}=\bigcup_{h \in I_{P}} K_{h}$.

Let us apply also the function $G_{x}=G_{\chi}$ which assigns a point $\chi_{h}$ as a distinguished point of $\chi\left(K_{h}\right)$ to each subsystem. Thereby, $G_{\chi}: \mathcal{M}_{\Pi} \rightarrow\left\{\left\{\chi_{h}\right\}\right\}$. We introduce also a mapping $\bar{\chi}: \mathcal{M}_{K} \rightarrow\left\{\left\{\chi_{h}\right\}\right\}$. Consequently, $G_{\chi}=\bar{\chi} \circ G_{K}$. In particular $G_{\chi}=G_{i}$ defines $\boldsymbol{X}_{h}$ as a distinguished point of $K_{h}$ in a reference configuration.

Let $H_{\chi h}=\left\{\chi_{m}, m \in I_{h}^{a}\right\}$ be a particular case of the set $H_{\alpha h}$ defined in the previous section. Then, we introduce the function $a_{h}: H_{\chi h} \rightarrow V_{a h}$ and $a:\left\{\left\{H_{\chi h}\right\}\right\} \rightarrow$ $\left\{\left\{a_{h}\left(\left\{\chi_{m}\right\}\right)\right\}\right\}$ as a function of kinematic dependence between subsystems defined here with the help of family $\mathcal{K}$. $V_{a h}$ is a linear space.

The function $\bar{\chi}$ assigns a set of discrete values of the field $\chi_{h}, h \in I_{P}$ to the body $\mathcal{B}$ with the help of the family $\mathcal{K}$. Similarly, the function $a$ assigns a set of discrete values of the field $a_{h}, h \in I$ to the body. However, $a_{h}$ depends on the finite set of values $\chi_{m}, m \in I_{h}^{a}$. The definition of the finite set is introduced with the help of the set of indexes $I_{h}^{a}$. This set in turn, contains indexes of elements of $\mathcal{K}$ which have influence on the value of $a_{h}$. Usually, they will be related to some neighbouring sets $K_{i}$ of $K_{h}$. Thus, the functions $\bar{\chi}$ and $a$ together can express nonlocal properties of the body.

Let $\bar{V}_{D}=\left\{\{\bar{\chi}, a\}:\{\bar{\chi}, a\}=\left\{\chi_{h}, a_{h}\right\}, h \in I_{P}\right\}$. Let us define the space $V_{\kappa}$ of deformation functions $\chi_{\kappa}$ of the body $\mathcal{B}$ with respect to a given configuration $\kappa$ as 
$V_{\kappa}=\left\{\chi_{\kappa}: \chi_{\kappa}=\lambda \circ \kappa^{-1}, \lambda, \kappa \in \mathcal{C}\right\}[6]$. Let furthermore, $\alpha_{\chi}: \bar{V}_{D} \rightarrow V_{\kappa}$ be a function and $\chi_{\kappa}^{\mathcal{K}}=\alpha\left(\left\{\chi_{h}, a_{h}\right\}\right)$, $\chi_{\kappa}^{\mathcal{K}}\left(\boldsymbol{X}_{h}\right)=\chi_{h}$.

Definition 3.2: The deformation function associated with the distinguished family of subbodies $\mathcal{K}$ is a function $\chi_{\kappa}^{\mathcal{K}}$ of the form $\chi_{\kappa}^{\mathcal{K}}=\alpha_{\chi}\left(\left\{\chi_{h}, a_{h}\right\}\right)$.

Definition 3.3: The motion of the body $\mathcal{B}$ associated with the family of sets $\mathcal{K}$ is a continuous map $\chi_{t}:[0, T] \rightarrow\left\{\chi_{\kappa}^{\mathcal{K}}\right\}$.

With the help of above definitions we have obtained deformation function and motion of the body in connection with the elementary dynamical system. This connection is expressed by means of functions $G_{K}$ and $G_{\chi}$.

We also introduce a function $\bar{T}$ on $\mathcal{K}$, which represents temperature, as $\bar{T}: \mathcal{K} \rightarrow R^{P}, \bar{T}\left(\left\{K_{h}\right\}\right)=\left\{T_{h}\right\}$. Let $I_{h}^{b} \subset I_{P}$ and $H_{T h}=\left\{T_{n}, n \in I_{h}^{b}\right\}$. Then, we introduce function $b_{h}$ by analogy to $a_{h}$ as $b_{h}: H_{T h} \rightarrow V_{b h}$ and $b:\left\{\left\{H_{T h}\right\}\right\} \rightarrow\left\{\left\{b_{h}\left(T_{n}\right)\right\}\right\}$.

Let us note that assignation of the value $T_{h}$ to the point $\chi_{h}$ in $\chi\left(K_{h}\right)$ is not so simple as defining $\chi_{h}$ which has direct geometrical interpretation. At this moment we do not discuss this problem precisely. Larger context for defining of temperature within continuum mechanics description will be discussed in what follows.

Let $\bar{V}_{T M}=\left\{\{\bar{T}, b\}:\{\bar{T}, b\}=\left\{T_{h}, b_{h}\right\}, h \in I_{P}\right\}$, $V_{T M}=\{T(\boldsymbol{x}): \boldsymbol{x} \in \chi(\mathcal{B})\}$. Let us consider a function $\alpha_{T}: \bar{V}_{T M} \rightarrow V_{T M}$ and $T^{\mathcal{K}}=\alpha_{T}\left(\left\{T_{h}, b_{h}\right\}\right)$.

Definition 3.4: The temperature field $T^{\mathcal{K}}$ associated with the distinguished family of subbodies $\mathcal{K}$ is the field obtained with the help of the function $\alpha_{T}$ as $T^{\mathcal{K}}=$ $\alpha_{T}\left(\left\{T_{h}, b_{h}\right\}\right)$.

The function $\alpha_{\chi}$ assigns a deformation function field $\chi_{\kappa}^{\mathcal{K}}$ to the set of its discrete values. The aim of this function is to introduce a continuous field $\chi$ on the body $\mathcal{B}$. Similar role plays the function $\alpha_{T}$ for $T$. Thus, the spaces of such fields $\operatorname{Im} \alpha_{\chi} \subset V_{\kappa}$ and $\operatorname{Im} \alpha_{T} \subset V_{T M}$ are finite-dimensional, where $\operatorname{Im} \phi$ means the image of a function $\phi$. Consequently, we have obtained continuum with finite-dimensional fields in relation to an elementary dynamical system.

\subsection{Balance of mass and energy for continuum mechanics}

We have introduced previously various assumptions related to collection of dynamical systems. They admit existence of functions $\tilde{m}, J_{\varphi i j}, c_{\varphi}, E_{\varphi}, W_{\varphi i j}, R_{\varphi}$ which introduce masses $m_{i}$, efflux of mass $J_{i j}$, source of mass $c_{i}$, efflux of energy $W_{i j}$ and source of energy $R_{i}$ accompanied by subsystems. Consequently, mentioned functions indexed here by $\varphi$ are referred directly to the elementary dynamical system.

We use these functions to reformulation of general form of balance of mass and energy equations defined for collection of dynamical system to the case of continuum.

Let $\tilde{m}: \mathcal{M}_{\Pi} \rightarrow\left\{\left\{m_{h}\right\}\right\}$ be mapping which determines a set of mass related to collection of dynamical systems. Let $\mathcal{M}_{M}=\left\{\left\{M_{h}\right\}\right\}$ and $M: \mathcal{M}_{K} \rightarrow \mathcal{M}_{M}$ be a function which determines masses assigned to $K_{h}$. We have also that $\pi_{h} \circ M(\mathcal{K})=M_{h}$, where $M_{h}$ is the total mass related to $K_{h} . M_{h}$ are defined by means of the relation $M \circ G_{K}=$ $i \circ \tilde{m}$, where $i$ is identity mapping. Consequently, a system of mass related to continuum is introduced by means of mapping $\tilde{m}$ defined on elementary dynamical system. Let $\mathcal{B}=\bigcup_{h} K_{h}, h \in I_{B}$, where $I_{B} \subset I_{P}$ is a set of indexes defining an arbitrary subbody $\mathcal{B}$ of the body also denoted by $\mathcal{B}$. Then, $M(\mathcal{B})=\sum_{h \in I_{B}} M_{h}$. Using this definition we obtain mass related to subbodies as a kind of measure defined on the body.

Energy accompanied by the elementary dynamical system is introduced by means of the function $E_{\varphi}: \mathcal{M}_{\Pi} \rightarrow$ $R^{P}$. Then, energy $\mathcal{E}: \mathcal{M}_{K} \rightarrow\left\{\mathcal{E}_{h}\right\}, \mathcal{E}_{h}=\pi_{h} \circ \mathcal{E}\left(\left\{K_{h}\right\}\right)$ assigned to each $K_{h}$, is defined by means of the relation $\mathcal{E} \circ G_{K}=i \circ E_{\varphi}$. As a result, we are able to define energy related to subbody as $\mathcal{E}(\mathcal{B})=\sum_{h} \mathcal{E}_{h}$. We assume further that $\mathcal{E}=E+\mathcal{T}$ is sum of internal energy and kinetic energy.

Source of mass $c_{\varphi}: \mathcal{M}_{\Pi} \rightarrow R^{P}$ and source of energy $R_{\varphi}: \mathcal{M}_{\Pi} \rightarrow R^{P}$ are defined now as $C: \mathcal{M}_{K} \rightarrow R^{P}$, $R: \mathcal{M}_{K} \rightarrow R^{P}$ by means of relations $C \circ G_{K}=i \circ$ $c_{\varphi}$ and $R \circ G_{K}=i \circ R_{\varphi}$. These quantities are defined for subbodies by means of expressions $C(\mathcal{B})=\sum_{h} C_{h}$, $R(\mathcal{B})=\sum_{h} R_{h}$

Efflux of mass $J_{\varphi i j}: \mathcal{M}_{\Pi} \rightarrow R$ and efflux of energy $W_{\varphi i j}: \mathcal{M}_{\Pi} \rightarrow R$ are defined as $J_{i j}: K_{i} \times K_{j} \rightarrow R$, where $J_{i j}$ is determined by means of $J_{i j} \circ\left(G_{K i} \times G_{K j}\right)=i \circ J_{\varphi i j}$ and $W_{i j}: K_{i} \times K_{j} \rightarrow R$, where we obtain $W_{i j}$ from $W_{i j} \circ\left(G_{K i} \times G_{K j}\right)=i \circ W_{\varphi i j}$.

Let us consider the boundary of the body $\partial \mathcal{B}=$ $\partial \bigcup_{h} K_{h}$. Then, $J(\partial \mathcal{B})=\sum_{i \in I_{B}, m \in I_{P}-I_{B}} J_{i m}$ and $W(\partial \mathcal{B})=\sum_{i \in I_{B}, m \in I_{P}-I_{B}} W_{i m}$.

We consider also $\partial \mathcal{B}_{s} \subset \partial \mathcal{B}$ which is defined as $\partial \mathcal{B}_{s}=$ $\bigcup_{h \in I_{s}}\left(\partial K_{h} \cap \partial \mathcal{B}\right), I_{s} \subset I_{B}$. There exists relation between $\partial \mathcal{B}_{s}$ and set of $J_{i m}$. We assume that pair of indexes $\{i, m\}$ is associated with $\partial \mathcal{B}_{s}$ if $\partial \mathcal{B}_{s}$ is a border between subsystems $i$ and $m$. Then, $J\left(\partial \mathcal{B}_{s}\right)=\sum_{i \in I_{s}, m \in I_{s i}} J_{i m}$.

With the help of introduced functions, the balance of mass Eq. (4) interpreted in terms of continuum is given by

$$
\dot{M}(\mathcal{B})+J(\partial \mathcal{B})-C(\mathcal{B})=0
$$

with the additional condition satisfied for arbitrary $\partial \mathcal{B}_{s} \subset \partial \mathcal{B}$

$$
J\left(\partial \mathcal{B}_{s}\right)=\bar{J}\left(\partial \mathcal{B}_{s}\right)
$$

The balance of energy Eq. (7) expressed in terms of continuum is assumed in the following form:

$$
\dot{E}(\mathcal{B})+\dot{\mathcal{T}}(\mathcal{B})+W(\partial \mathcal{B})-R(\mathcal{B})=0,
$$

with the additional condition satisfied for arbitrary $\partial \mathcal{B}_{s} \subset \partial \mathcal{B}$

$$
W\left(\partial \mathcal{B}_{s}\right)=\bar{W}\left(\partial \mathcal{B}_{s}\right) .
$$

Neglecting at the moment detailed representations of introduced below quantities, we formulate also the second law of thermodynamics as a supplementary postulate. This is given with the help of the balance entropy expressed as 


$$
P(\mathcal{B})=\dot{S}(\mathcal{B})+H(\partial \mathcal{B})-N(\mathcal{B}) \geq 0,
$$

where $S$ is entropy, $H$ is efflux of entropy and $N$ stands for source of entropy. $P$ represents production of this quantity.

Above introduced balance of mass and energy equations should be considered as framework for determination of a continuum skeletal dynamical system. This can be done when we apply particular representations of quantities which appear in (18)-(22). The continuum skeletal dynamical system is then element of dimensional reduction procedure applied to an elementary dynamical system. In particular this elementary dynamical system can represent molecular dynamics equations and by this can create foundations for the continuum description.

\subsection{Comments on role of molecular dynamics within unified mechanics of materials}

Discussed in previous section concept of multiscale modelling called collection of dynamical systems with dimensional reduction is aimed at integration of various scale processes within one theoretical framework. However within this approach we should indicate preferred scales for corresponding unified description.

The first distinguished scale is the atomic scale and by this description by molecular dynamics method. On this level we can describe interactions between atoms by corresponding potentials. Various phenomena in materials has to be discussed just on atomic level. Let us mention mechanisms of various type reorganization of atomic arrangements. Recrystallization of material during slip plasticity induced by temperature changes can be seen as an example of such a reorganization.

Let us elucidate role of molecular dynamics within collection of dynamical systems with dimensional reduction by considering some particular methods of modelling.

Physical systems frequently can be characterized by variables of two types. The first one is related to slowly varying and the second one to quickly varying processes. Well known example is related to a system with deformation and temperature considered within continuum mechanics.

Having this in mind we illustrate here a general concept of creating of foundations for continuum mechanics basing on such a decomposition. Consequently, let us introduce a special case of $\pi_{T}=\left\{\pi_{S T}, \pi_{Q T}\right\}$ in which two parts related to slowly and quickly varying variables are discriminated. As a result of this $\boldsymbol{d}(t)=\pi_{T}(\varphi(t))$ can be expressed as $\boldsymbol{d}=\left\{\boldsymbol{d}_{S}, \boldsymbol{d}_{Q}\right\}=\left\{\pi_{S T}(\boldsymbol{\varphi}), \pi_{Q T}(\boldsymbol{\varphi})\right\}$.

Let us assume also that the elementary dynamical system is a Hamiltonian dynamical system which takes the form

$$
\frac{\mathrm{d} \boldsymbol{q}_{i}}{\mathrm{~d} t}=\boldsymbol{v}_{i}, \quad m_{i} \frac{\mathrm{d} \boldsymbol{v}_{i}}{\mathrm{~d} t}=-\frac{\partial H}{\partial \boldsymbol{q}_{i}}+\boldsymbol{f}_{i},
$$

where $i \in I_{N}=\{1,2, \ldots, N\}, H$ is a Hamiltonian and $\boldsymbol{f}_{i}$ is a force acting on $i$-th material point. Let $\boldsymbol{q}=\left\{\boldsymbol{q}_{i}\right\}$, $\boldsymbol{v}=\left\{\boldsymbol{v}_{i}\right\}$. Then, variables of our EDS takes the form $\boldsymbol{\varphi}=\{\boldsymbol{q}, \boldsymbol{v}\}$.
Such an option of the elementary dynamical system suggests possibility of application of molecular dynamics calculations for creation of physical foundations for continuum model. Consequently, we assume in general that physical foundations for more averaged models, in particular continuum models, are better expressible in smaller scale. This assumption seems to be natural since fundamental physics is related directly to elementary particles and atoms. On the other hand, currently applied equations of more averaged continuum models are based usually on assumptions related to considerable averaged processes. However, this averaging is frequently based on intuition related to corresponding scale or experimental data related to averaged processes. Then, we are not aware which processes are lost during this averaging by intuition. All this justifies general tendency to obtain better physical foundations following from atomistic models.

Let us take a set of time instants $t_{0}<t_{1}<\ldots<t_{K}$ which belong to the time interval $T=\left[t_{0}, t_{0}+T\right]$, $t_{K}=t_{0}+T$, and let $I_{K}=\{0,1, \ldots, K\}$. By means of these instants we divide the time interval into the sum $T=\bigcup_{k} T_{k}, T_{k}=\left[t_{k-1}, t_{k}\right], k=1, \ldots, K$. Then, for each $k$ we can calculate the value of $\tilde{\boldsymbol{q}}_{k}$ as

$$
\tilde{\boldsymbol{q}}_{k}=\frac{1}{T_{k}} \int_{T_{k}} \boldsymbol{q}(t) \mathrm{d} t .
$$

A value $\tilde{\boldsymbol{q}}_{0}=\boldsymbol{q}\left(t_{0}\right)$ is assigned to $k=0$. With the aid of sequence of values $\left\{\tilde{\boldsymbol{q}}_{k}\right\}, k \in I_{K}$ we can generate a function $\tilde{\boldsymbol{q}}(t)=I_{q}\left(\left\{\tilde{\boldsymbol{q}}_{k}\right\}\right)$, where $I_{q}$ is an approximation procedure. Now, we are able to decompose the variable $\boldsymbol{q}(t)$ into two summands

$$
\boldsymbol{q}(t)=\tilde{\boldsymbol{q}}(t)+\delta \boldsymbol{q}(t) .
$$

Thus, $\tilde{\boldsymbol{q}}(t)$ represents the slowly varying part of $\boldsymbol{q}(t)$ and $\delta \boldsymbol{q}(t)$ its rapidly varying part.

The question is what properties should the approximation procedure $I_{q}$ have in order to describe real slowly varying process in accordance with the introduced set $\left\{\boldsymbol{q}_{k}\right\}$. Slowly varying variable should gradually change its values between given points. This fact should be expressed by possible small values of the second time derivatives. Consequently, the approximation procedure $I_{q}$ could be defined as a process of finding a function $\tilde{\boldsymbol{q}}(t)$ which satisfy the following set of properties:

$$
\left\{\begin{array}{l}
I_{q}\left(\left\{\tilde{\boldsymbol{q}}_{k}\right\}\right)=\tilde{\boldsymbol{q}}(t), \\
\sup _{t \in T}\left(\left|\frac{\partial^{2} \tilde{\boldsymbol{q}}}{\partial t^{2}}\right|\right) \rightarrow \inf \\
\tilde{\boldsymbol{q}}\left(\tau_{k}\right)=\tilde{\boldsymbol{q}}_{k}, \tau_{k}=0.5\left(t_{k-1}+t_{k}\right), \\
\tilde{\boldsymbol{q}}(t) \in C^{n}, n \geq 2, t \in T
\end{array}\right.
$$

It should be expected also that $\int_{T_{k}}(\boldsymbol{q}-\tilde{\boldsymbol{q}}) \mathrm{d} t=\int_{T_{k}} \delta \boldsymbol{q} \mathrm{d} t \approx$ 0 , where $" \approx "$ means equality with an admissible error. As a result of this decomposition we are able to consider $\dot{\boldsymbol{q}}(t)=\dot{\tilde{\boldsymbol{q}}}(t)+\delta \dot{\boldsymbol{q}}(t)$. Decomposition defined by (26) has been realized by means of finite element approximation in [7].

Let $A_{t}(\{\boldsymbol{q}, \dot{\boldsymbol{q}}\}(t))=\{\tilde{\boldsymbol{q}}, \delta \boldsymbol{q}, \dot{\tilde{\boldsymbol{q}}}, \delta \dot{\boldsymbol{q}}\}(t)$. Let us introduce further special kinds of variables. Let they be 
denoted by $\boldsymbol{d}=\left\{\boldsymbol{d}_{S}, \boldsymbol{d}_{Q}\right\}, \boldsymbol{d}_{S}=\left\{\boldsymbol{d}_{S C}, \boldsymbol{d}_{S \xi}, \boldsymbol{d}_{S V}\right\}$ and $\boldsymbol{d}_{Q}=\left\{\boldsymbol{d}_{Q C}, \boldsymbol{d}_{Q \theta}, \boldsymbol{d}_{Q V}\right\}$, where $\boldsymbol{d}_{S C}, \boldsymbol{d}_{Q C}$ are called configurational variables, $\boldsymbol{d}_{S V}=\dot{\boldsymbol{d}}_{S C}, \boldsymbol{d}_{Q V}=\dot{\boldsymbol{d}}_{Q C}$. It is admitted that not all $\dot{\boldsymbol{d}}_{S C}, \dot{\boldsymbol{d}}_{Q C}$ are present in set of variables $\boldsymbol{d}_{S}, \boldsymbol{d}_{Q}$. Some of them can be neglected. $\boldsymbol{d}_{S \xi}$, $\boldsymbol{d}_{Q \theta}$ are internal state variables.

Taking into account above decompositions we assume that there exists mappings $\pi_{S}$ and $\pi_{Q}$ which gives $\left\{\boldsymbol{d}_{S C}, \boldsymbol{d}_{S \xi}\right\}=\pi_{S} \circ A_{t}(\boldsymbol{\varphi}(t))$ and $\left\{\boldsymbol{d}_{Q C}, \boldsymbol{d}_{Q \theta}\right\}=$ $\pi_{Q} \circ A_{t}(\varphi(t))$. Let $D_{S t}\left(\left\{\boldsymbol{d}_{S C}, \boldsymbol{d}_{S \xi}\right\}\right)=\boldsymbol{d}_{S}$ and $D_{Q t}\left(\left\{\boldsymbol{d}_{Q C}, \boldsymbol{d}_{Q \theta}\right\}\right)=\boldsymbol{d}_{Q}$. Then the form of $\pi_{T}$ is determined by

$$
\begin{aligned}
& \pi_{T}=\left\{\pi_{S T}, \pi_{Q T}\right\}= \\
& \quad\left\{D_{S t} \circ \pi_{S} \circ A_{t}, D_{Q t} \circ \pi_{Q} \circ A_{t}\right\} .
\end{aligned}
$$

We introduce also the map $\pi\left(\boldsymbol{\varphi}_{0}\right)=\boldsymbol{d}_{0}$ which depends on $\pi_{T}$ by assumption that $\pi\left(\boldsymbol{\varphi}\left(t_{0}\right)\right)=\pi\left(\pi_{T}(\boldsymbol{\varphi}(t)), \boldsymbol{\varphi}\left(t_{0}\right)=\right.$ $\left.\varphi_{0}\right)$. By means of $\pi$ initial conditions can be transferred between scales.

Similar SQ-decomposition can be carried out for forces $f$. To this end we calculate the following time averaged quantities:

$$
\tilde{\boldsymbol{f}}_{k}=\frac{1}{T_{k}} \int_{T_{k}} \boldsymbol{f}(t) \mathrm{d} t .
$$

Then, we have $\tilde{\boldsymbol{f}}(t)=I_{f}\left(\tilde{\boldsymbol{f}}_{k}\right)$ with the help of an approximation procedure $I_{f}$. Finally, we obtain the decomposition

$$
\boldsymbol{f}(t)=\tilde{\boldsymbol{f}}(t)+\delta \boldsymbol{f}(t) .
$$

Let $B_{t}(\boldsymbol{f}(t))=\{\tilde{\boldsymbol{f}}(t), \delta \boldsymbol{f}(t)\}$ and $\pi_{S f} \circ B_{t}(\boldsymbol{f}(t))=\overline{\boldsymbol{f}}_{S}$, $\pi_{Q f} \circ B_{t}(\boldsymbol{f}(t))=\overline{\boldsymbol{f}}_{Q}$. Finally, we obtain the general form of the map

$$
\pi_{f T}=\left\{\pi_{S f} \circ B_{t}, \pi_{Q f} \circ B_{t}\right\} .
$$

In general it is expected that mappings $\pi_{T}$ and $\pi_{f T}$ are not entirely independent. Structure of $\pi_{T}$ and operator $\mathcal{L}_{T}$ should have influence on kind of dimensional reduction applied to $f$. Establishing of possible dependence between $\pi_{T}, \pi_{f T}$ is very complicated in general case. We postulate merely that there exist relation $\mathcal{R}_{L}\left(\pi_{T}, \pi_{f T}\right)$ which join considered two mappings. Consequently, it is assumed that in the case of SQ decomposition $\mathcal{R}_{L}=I$ or in other words, the same procedures are represented by $\pi_{T}$ and $\pi_{f T}$.

Accordingly, we have obtained the following decomposition of variables of the new dynamical system: $\boldsymbol{d}=\left\{\boldsymbol{d}_{S}, \boldsymbol{d}_{Q}\right\}=\left\{\boldsymbol{d}_{S C}, \boldsymbol{d}_{S \xi}, \boldsymbol{d}_{S V}, \boldsymbol{d}_{Q C}, \boldsymbol{d}_{Q \theta}, \boldsymbol{d}_{Q V}\right\}$.

We have already introduced two kinds of variables $\chi_{h}$ and $T_{h}$ for our continuum with finite-dimensional fields. It means that SQ-decomposition is accepted in continuum description. Indeed, $T_{h}$ represents averaged properties of quickly varying processes and $\chi_{h}$ the slowly varying ones.

We can introduce the following interpretation of introduced variables: $\boldsymbol{d}_{S C}=\left\{\chi_{h}\right\}, \boldsymbol{d}_{S V}=\left\{\dot{\chi}_{h}\right\}, \boldsymbol{d}_{S \xi}=\left\{\eta_{h}\right\}$ as internal state variables, $\boldsymbol{d}_{Q C}=\left\{T_{h}\right\}, \boldsymbol{d}_{Q \theta}=\emptyset$, $\boldsymbol{d}_{Q V}=\emptyset$. In two last cases we have neglected internal state variables related to quickly varying processes and time derivative of temperature $T_{h}$.

It is possible to deduce using also form of EDS (23) that the balance of energy equation (20) can be expressed in the form [4]:

$$
\begin{aligned}
& \dot{E}(\mathcal{B})+\dot{\mathcal{T}}(\mathcal{B})+W_{S}(\partial \mathcal{B})+W_{Q}(\partial \mathcal{B})-R_{S}(\mathcal{B}) \\
& \quad-R_{Q}(\mathcal{B})=0 .
\end{aligned}
$$

We can assume also that balance of mass equation subsequently applied takes the simple form $\dot{M}_{h}=0$.

These equations lead to determination of continuum skeletal dynamical system by means of application of particular representation of quantities in (31). Consequently we see an example of using of molecular dynamics method as an elementary dynamical system which is next applied to determination of reduced dimensionally dynamical system representing continuum description.

Molecular dynamics simulations lead to gathering of considerable amount of data. As a result of this it is difficult to interpret and manipulate such a data. Collection of dynamical systems with dimensional reduction can transform obtained by molecular dynamics simulations data into dimensionally reduced models of mechanics of materials. This shows that possible role of molecular dynamics method within collection of dynamical system with dimensional reduction is to produce data by numerical simulation and next coin on this basis reduced dimensionally models. Then the reduced models can be seen as containers of these data represented by constants of the reduced models.

Above discussion accentuates also difference in this approach and statistical mechanics approach where physical basis for continuum mechanics can also be created. In our case all models are deterministic. Method of averaging of solutions defines temperature for instance as related to averaging of quickly varying processes. Thereby, we see that precise definition of temperature on this way can be difficult but is important. This is not stochastic averaging.

Let us note that temperature should be well defined also in case of deterministic models. Stochasticity in this case is related rather to initial conditions. However it is imaginable that we know such initial conditions precisely. Then, temperature should be also well defined. Thereby, stochasticity should not be necessary condition for definition of temperature. Let us also note that formulation of stochastic approach in case of multiscale method represented by collection of dynamical systems has been discussed in [8].

\subsection{Comments on role of nanoscale models within unified mechanics of materials}

The second distinguished scale of modelling within our striving towards unified mechanics of materials following directly after the atomic scale is the nanoscale.

Mechanisms of inelastic deformation are frequently related to nanoscale description. Let us mention mechanisms of martensitic transformation leading to considerable strain of material or mechanisms of slip plasticity. 
In the first case we have to describe mechanisms of motion of single interfaces between austenite and martensite or between various martensite variants. In the second case we have to consider single slip surfaces.

Important role is designed to nanoscale description of phenomena in materials. Let us notice that such models are rather complex. By this they are usually not convenient for direct engineering applications in similar way as macroscopic models. This makes difficult to find justification for development of nanoscale models. However, by providing above discussed multiscale method of modelling we determine context for development of nanoscale models. They should play role of descriptions related to intermediate scale between atomic one and more averaged engineering models.

Hitherto in literature we have to do with statistical mechanics which is applied to transition from atomic models to more averaged ones. However, this transition is difficult when considerable number of phenomena happens in intermediate scales. This is seen as obstacle against wide application of statistical mechanics in engineering. Therefore indicating nanoscale models as models of intermediate scale, where transition between various scales is carried out by method called here dimensional reduction, can make possible wider application of descriptions based on atomic scale.

We tend towards unified mechanics of materials. Therefore we should have at our disposal also possibility of unified description of various phenomena on the same nanoscale level. In literature we encounter models which can be interpreted as nanoscale ones [9-11]. They are based on Landau theory where free energy is determined by means of polynomials. This approach is not too convenient for unification of various phenomena. In case of more complex processes the free energy has to be defined by means of polynomials with very high degree. However it is difficult to carry out physical interpretation of introduced models with the aid of such polynomials.

In order to avoid this difficulty one introduces method which determines graph of the free energy for nanoscale description as fibre manifold spanned on geometrical skeleton which has good physical interpretation $[4,12]$. Let us mention shear systems for martensitic transformation or slip systems for slip plasticity with single slip surfaces, space distribution of habit planes for martensitic transformation in crystal structure for instance as examples of elements of such a geometrical skeleton.

This method of determination of the free energy function is applied in elaboration of nanoscale description of martensitic transformation [12-16], nanoscale description of slip plasticity [17] or nanoscale model of fracture [18].

Free energy defined by means of fibre manifold allow us to introduce unified description of martensitic transformation and slip plasticity [19] and also unify this description with description of fracture [4]. Discussion of whole methodology leading to unified description in mechanics of materials has been carried out in [20].
Summarizing, by above discussion we accentuate particular role which is assigned to nanoscale models on way leading to unified mechanics of materials. We have elaborated methodology of unifying description on the same nanoscale level. On the other hand nanoscale models are seen as segment of unified multiscale description by collection of dynamical systems where they are a bridge between atomic scale models and more averaged continuum models.

\subsection{Remarks on descriptions with various types of dimensional reductions}

We have mentioned in introduction to collection of dynamical systems that joining of various types of models obtained by various types of dimensional reductions procedures is important for unification of theoretical description.

Let us note that smaller scale models have more homogeneous description and transition to another model is less frequent. Let us mention description by molecular dynamics at atomic level. Then all phenomena can be described by the same equations as a matter of fact. We neglect at this moment possibility of various descriptions at this level.

Then after the dimensional reduction procedure towards nanoscale models we can obtain various models. Let us mention physically nonlinear elasticity and discussed previously martensitic transformation, slip plasticity or fracture. The question is how we can define critical conditions for change of description during numerical simulations.

Within nanoscale models critical conditions are determined by means of the free energy function $[4,17,19]$. Boundary of elastic region for nonlinear elasticity defined within the free energy determines conditions for initiation of transition to new martensite variant, initiation of slip on a given slip surface or initiation of a crack. After exceeding of the critical conditions additional equations can be activated for further description of evolution of material. By this fact unificational role of the free energy is accentuated. In this place important role of method of determination of the free energy function as fibre manifold is also accentuated.

Critical conditions for larger scale models have to be determined with the aid of analysis of particular dimensional reductions methods.

\subsection{On concept of design of structures and processes in relation to various scales}

We should comment why the multiscale description which should be considered in a future as unified description of processes in materials creates appropriate environment for the design processes.

Let us note that the design process could be related to arbitrary aspects expressed by the model. Therefore, universality of such a design needs unified description of all phenomena. It explains why we tend just towards unified description. On the other hand, design criteria 
can be related to properties expressed in various scales. Therefore multiscale description seems to be appropriate.

Let us express the design task in the following general form:

$$
\left\{\begin{array}{l}
\mathcal{J}(x, u) \rightarrow \text { inf, } \\
\dot{x}=A(x, u), \\
x\left(t_{0}\right)=x_{0} \in X, u \in U .
\end{array}\right.
$$

expressed above task of design contains functional $\mathcal{J}$, equations describing evolution of material associated with variables $x$ in general, the set $X$ and the set $U$ containing parameters $u$. Design criteria are expressed by the functional $\mathcal{J}$ and sets $X$ and $U$.

We decide which scale is applied for description of processes. If the design is associated with organization of atoms then molecular dynamics equations could be appropriate. The set $X$ could be responsible for arrangement of atoms as well as their kinds expressible within initial conditions. However other design criteria could be related to various scales.

The functional $\mathcal{J}$ could represent elastic constant for instance as related to considerably larger scale. Parameters $u$ also could represent various properties related to various scales. They could be calculated by means of methods of transition between various scale models. Consequently the dimensional reduction procedure would be taken into account in defining the functional $\mathcal{J}$ and also defining role of parameters $u$ in this functional.

Above discussed kind of thinking manifests tendency towards making design of structures and processes in varios scales as systematic procedure.

\section{Concept of theory directed to determination of source of precision for nanotechnological processes}

\subsection{Vacuum medium mechanics as theory which} indicates source of precision for small scale processes

Small scale processes are associated with dynamics to larger degree than large scale processes where we can consider even statics. Observing biological processes in cell corresponding to nanotechnology such as protein synthesis or repair of DNA for instance we see large precision within processes associated with considerable dynamics. This suggests that we have to do there with an attractor representing just dynamical properties, which is responsible for self-organization on the most fundamental level and manifested in corresponding molecular systems. This self-organization is seen in this case as maintaining high precision of processes.

The question is which theory is able to indicate this attractor. Let us note that persistence of life indicates that this attractor is associated with considerable power and accidental nonlinearities are rather not able to define it.

Quantum mechanics is constructed initially as linear theory [21, 22]. Sometimes it is modified to a nonlinear theory. Quantum electrodynamics has structure close to quantum mechanics $[22,23]$. Furthermore, mentioned theories are based on geometry to large degree. Therefore description of dynamics seems to be of secondary aim considered during construction of these theories. System of fundamental notions applied to these theories is rather poor in order to describe mechanism of motion of elementary particles. Let us mention in this place that these theories consider point like-particles.

Observed nanotechnological processes in cell, considered as open with respect to matter and energy, suggest that excited states are important in these processes. On the other hand, excited states in long molecules seem to be rather nonstationary one. This does not fit entirely to quantum mechanical description where excited states are associated with an eigenvalue of an operator and are similar by this to stationary ones.

Above discussion suggests that quantum mechanics or quantum electrodynamics do not describe appropriately dynamics on the most fundamental level and are not able to indicate attractor governing self-organization within molecular processes in cell.

The question is which theory is able to do it. Above discussion shows that we need a fundamental theory of elementary particles which should describe an attractor. Furthermore, molecular systems in single cell are energetically open systems. This means that excited states have to be modelled also in the context of self-organization process.

Let us note that long lasting evolution leads to homeothermality. This is real experiment carried out by nature. It indicates that conjugation of electronic and nuclei motion in molecular systems should be described with appropriate quality. All this induces serious requirements for our fundamental theory.

In order to construct fundamental theory describing so many aspects for low energy elementary particles we should use sufficiently rich set of premises. Furthermore, we should have confidence in such a theory. In other words it is difficult to separate low energy physics from high energy physics since both descriptions should be consistent. Let us notice that many experimental results which would be taken into account as premises follow from high energy accelerator experiments. In order to obtain still more profound system of premises and tests of our theory we should also use experimental results which are obtained by nature in cosmological scale. Contradiction of our theory with cosmological results would decrease confidence in our theory having in mind its fundamental and universal aspects.

In order to maintain mentioned consistency we should construct in fact a fundamental theory which is universal. Then, our confidence in such a theory would be appropriate.

Consequently, in order to construct universal theory we should organize first a plane for unification of all four elementary particle interactions instead of constructing directly models of separate interactions. In order to model better dynamical processes we should organize more elementary level for doing assumptions of our theory. This 
level should be placed even below the elementary particles level.

The last statement means that elementary particles should not be point-like particles since point-like approach means in fact averaging of a real particle properties to whole particle avoiding description of its internal dynamics. Thereby particles should be modelled as extended and have its own internal dynamics which would be able to explain mechanisms of motion of these particles. Importance of description of mechanisms of motion follows from observed difficulties with explanation of role of mass in elementary particle physics. It also means that just dynamics of processes is treated superficially by current theories. In order to satisfy above requirements we introduce vacuum medium mechanics [24-31] where vacuum medium is considered as four-component. At first stage of development of such a theory we construct qualitative model of all important and observed phenomena associated with all four elementary particle interactions. This step is important in order to determine system of fundamental notions useful for unification of all mentioned elementary particle interactions. Placement of postulates within vacuum medium give a chance for better description of dynamics. Elementary particles appear then as extended, not point-like objects with internal dynamics. Within vacuum medium mechanics we are able to characterize attractor responsible for selforganization of molecular systems. This leads to determination of mechanisms of evolution of biological systems based on the most fundamental level of description as well as characterizing source of precision necessary for nanotechnological processes.

\subsection{Assumptions related to the vacuum medium structure}

The vacuum medium mechanics is discussed in several papers [24-31]. We introduce here main assumptions related to the vacuum medium in order to provide context for further discussion.

Various cosmological investigations indicate that the space of the Universe is flat. Furthermore, investigations of background radiation show existence of a reference configuration with respect to radiation in our space.

Having in mind above remarks we identify our medium with three-dimensional space $E^{3}$, where we introduce a Cartesian coordinate system $\boldsymbol{X}=\left\{X_{i}\right\}, i=1,2,3$, $\boldsymbol{X} \in E^{3}$. We assume furthermore that behaviour of our medium does not induce any changes of introduced geometry at this stage of our considerations.

The vacuum medium is considered as a mixture of four components [24] joined within an elementary unit identified at this moment with a point of space. Motivations for assuming four components follows from observation that the Maxwell equations exhibit a symmetry with respect to electric and magnetic field. This induces considering at least two components. However, creation of electron-positron pair indicates that a separation of components is associated with electric field only. Therefore, two components are assigned to electric field. By anal- ogy to observed symmetry between electric and magnetic fields two other components are also assigned to the magnetic field.

It is further assumed here that these components constitute four-component elementary units which create a stable medium for low energy states. In the continuum description applied here the elementary units correspond to points of space. However, in particular cases we can also assume that elementary units have a finite size which is more natural and convenient in our considerations.

We introduce densities which represent an amount of component related to a volume which can be discussed owing to the introduced coordinate system. Thus, we assume that $\varrho_{v}, \varrho_{\bar{v}}, \varrho_{w}, \varrho_{\bar{w}}$ and $\varrho$ stand for densities of the components and the density of the united media, respectively. We have then

$$
\varrho_{v}+\varrho_{\bar{v}}+\varrho_{w}+\varrho_{\bar{w}}=\varrho .
$$

Symbols of these densities will be applied also as names for corresponding to them media.

State of each elementary unit is described by displacements or a kind of polarization of discussed components within units. They are represented by vectors $\boldsymbol{v}, \overline{\boldsymbol{v}}, \boldsymbol{w}$, $\overline{\boldsymbol{w}}$. We assume that two pairs of the components are discriminated by special interactions. Components within each pair are able to move with respect to each other. As a result, we can reduce in some cases the number of variables by introducing the new ones: $\boldsymbol{u}=\boldsymbol{v}-\overline{\boldsymbol{v}}$ and $\boldsymbol{q}=\boldsymbol{w}-\overline{\boldsymbol{w}}$. At this moment it is also assumed that $\overline{\boldsymbol{v}}=-\boldsymbol{v}, \overline{\boldsymbol{w}}=-\boldsymbol{w}$.

Considering state of the elementary unit we come to a natural question. To what degree such a polarization has a geometrical representation associated with classical displacements.

It seems reasonable that direction in space could be well defined within the elementary unit. Indeed, our intuition related to geometrical interpretation of space follows from straight propagation of light. In discussed model propagation of light is connected with transfer of interactions between elementary units. Therefore, it seems to be reasonable that such interactions determine direction within the elementary unit.

Interpretation of the polarization as displacements is perhaps not entirely convenient. Length of vector representing polarization should be in accordance with a size of the elementary unit which imposes constraints on degree of polarization. Therefore, we introduce a relation between degree of polarization and assigned to it displacements. We do this by functions

$$
\begin{aligned}
\boldsymbol{v}_{G} & =\mathcal{G}_{v}(\boldsymbol{v}), \overline{\boldsymbol{v}}_{G}=\mathcal{G}_{\bar{v}}(\overline{\boldsymbol{v}}), \\
\text { and } & \\
\boldsymbol{w}_{G} & =\mathcal{G}_{w}(\boldsymbol{w}), \overline{\boldsymbol{w}}_{G}=\mathcal{G}_{\bar{w}}(\overline{\boldsymbol{w}}) .
\end{aligned}
$$

The functions $\mathcal{G}_{v}, \mathcal{G}_{\bar{v}}, \mathcal{G}_{w}, \mathcal{G}_{\bar{w}}$ assign geometrical displacements to vectors describing state of the elementary unit as vectors having the same directions.

Let $|\boldsymbol{v}|$ be length of the vector $\boldsymbol{v}$. Then, structure of the function $\mathcal{G}_{v}$ can be expressed as $\mathcal{G}_{v}(\boldsymbol{v})=\tilde{\mathcal{G}}_{v}(|\boldsymbol{v}|) \boldsymbol{n}_{v}$, where $\boldsymbol{n}_{v}=\boldsymbol{v} /|\boldsymbol{v}|$. 
Assignation of displacements to each kind of polarization enables incorporating of results of classical continuum mechanics to our methods of modelling. Consequently, we can introduce interpretation that each component deforms separately during interactions with the remaining ones. We are able to describe such a deformation by means of the deformation function which is considered in continuum mechanics [6]. This is formally possible since we have introduced the coordinate system.

Consequently, deformation of each component is described by deformation functions $\boldsymbol{x}_{v}=\chi_{v}(\boldsymbol{X}), \boldsymbol{x}_{\bar{v}}=$ $\chi_{\bar{v}}(\boldsymbol{X}), \boldsymbol{x}_{w}=\chi_{w}(\boldsymbol{X}), \boldsymbol{x}_{\bar{w}}=\chi_{\bar{w}}(\boldsymbol{X})$, where $\boldsymbol{X}$ are points of a reference configuration. As a result we can consider displacements determined by means of (34) and (35) in connection with the deformation function by expressions

$$
\begin{aligned}
& \boldsymbol{v}_{G}=\boldsymbol{x}_{v}-\boldsymbol{X}, \quad \overline{\boldsymbol{v}}_{G}=\boldsymbol{x}_{\bar{v}}-\boldsymbol{X}, \\
& \boldsymbol{w}_{G}=\boldsymbol{x}_{w}-\boldsymbol{X}, \quad \overline{\boldsymbol{w}}_{G}=\boldsymbol{x}_{\bar{w}}-\boldsymbol{X} .
\end{aligned}
$$

The reference configuration can be interpreted as describing nonexcited, equilibrium vacuum medium.

The variables $\boldsymbol{u}$ and $\boldsymbol{q}$ are identified with the vector of the electric field intensity $\boldsymbol{E}$ and the magnetic induction vector $\boldsymbol{B}$, respectively. Propagation of $\boldsymbol{u}$ and $\boldsymbol{q}$ through the vacuum medium is interpreted as electromagnetic wave.

We also introduce a displacement $\boldsymbol{h}_{G}$ in the elementary unit corresponding to gravitational field $\boldsymbol{h}$ by

$$
\boldsymbol{h}_{G}=\mathcal{G}_{h}(\boldsymbol{h}) \text {. }
$$

We do not interpret this displacement precisely. It represents an internal state of the elementary unit. Perhaps it is related to deformation of such a unit. Perhaps it represents a reorganization having vectorial character. We can also admit a fifth component into considerations. However, at this moment we have not sufficient premises for introduction of interpretations.

In the paper [24] we have assumed that the medium $\varrho$ can be decomposed into the sum $\varrho=a+b$ for higher energy, where

$$
\text { and }
$$$$
a=\varrho_{v}+\frac{1}{2}\left(\varrho_{w}+\varrho_{\bar{w}}\right)
$$

$$
b=\varrho_{\bar{v}}+\frac{1}{2}\left(\varrho_{w}+\varrho_{\bar{w}}\right) .
$$

This decomposition appears as a result of attaining by $\boldsymbol{u}$ a critical value $\boldsymbol{u}^{*}$ characteristic for the discussed medium.

Relations (38) and (39) can be understood as describing change of densities during separation of component process. However, they should be understood less formally rather as a balance of amount of corresponding components denoted also as densities. This is so since we do not analyse here any change of volumes. Furthermore we assume in what follows that volume corresponding to $a$ and $b$ is considerably smaller than that one corresponding to $\varrho$. Thereby, the relations (38) and (39) are devoted at this stage of considerations to accentuate that a change of structure within component organization happens. We do not understand of this change too precisely yet. However, by mentioning of the relations (38) and (39) we obtain a bridge-head for further analysis of mechanisms of separation of components.

Components $a$ and $b$ create medium of electron and positron respectively when they rotate. In such a case the components are separated from the remaining part of the medium by a discontinuity surface. Then, motion of this surface determines of motion of electron or positron.

We also admit rotation of a part of the $\varrho$-medium separated by a discontinuity surface from remaining part of stable $\varrho$-medium. Such a state is identified with neutrino and motion of the discontinuity surface describes motion of the neutrino. This motion is not associated with separation of components. Therefore motion of neutrino is not associated with any wave function.

We assume that each component $\varrho_{v}, \varrho_{\bar{v}}, \varrho_{w}, \varrho_{\bar{w}}$ considered separately attracts its own elements. Components $a$ and $b$ have the same property. Attraction between various kinds of components takes place for sufficiently small energy which leads to formation of the elementary units. Components $a$ and $b$ after separation also exhibit attraction which can lead, for some conditions, to recovering of the elementary units and thereby the four-component vacuum medium structure.

We could consider several energetic levels with different kinematics. The lowest energy is connected with displacements $\boldsymbol{u}$ and $\boldsymbol{q}$ only. Higher energy levels are associated with elementary particles. Consequently, electron and positron are viewed as rotating $a$ and $b$-media separated from $\varrho$ by a discontinuity surface [24]. This separation happens when a critical value $\boldsymbol{u}=\boldsymbol{u}^{*}$ is attained by the electric field. Interactions between particles and electromagnetic field are determined with the help of boundary conditions given on the discontinuity surface.

One also admits coexistence of components $a, b$ and $\varrho$ in the same point of space. Such a state is considered as a nonequilibrium state of the vacuum medium and has to relax to a lower energy level. In particular when electron moves then motion of the separation surface is associated with production of an excess of $b$ before the particle and transferring of $a$ into the particle as well as stopping part of $a$ behind the electron. This induces just nonequilibrium distribution of components around the particle. Consequently $\{a, b, \varrho\}(\boldsymbol{X})$ associated with moving particle is interpreted as the wave function. Problem of evolution of such a nonequilibrium distribution of components was discussed in [26].

Introduced above notions lead to a continuum description of the vacuum medium related to a scale considerably smaller than size of particles such as electron or neutrino. Therefore, the particles are seen by the model as extended particles.

\subsection{General characterization of attractor} considered within vacuum medium mechanics

Vacuum medium mechanics is formulated with the aim of improving description of dynamics of elementary particles. In this approach considerably extended system of fundamental notions is introduced in comparison with other theories of elementary particles. These fundamen- 
tal assumptions are related directly to vacuum medium and elementary particles, their charge, spin and mass appear as consequence and are not fundamental notions. As a result of this dynamics of processes related to elementary particles can be described in larger context.

The chain state of particles with the same charge is important type of interactions which does not appear in quantum mechanics and is described within vacuum medium mechanics $[26,28]$. One indicates that this dynamical state is responsible for self-organization just in molecular systems [28].

Electron within vacuum medium mechanics is considered as extended particle. Full description of electron is a complicated task and its theory is not finished yet within vacuum medium mechanics. However, it is shown that important properties of electron are expressible within vacuum medium mechanics. Let us mention here description of waving of component distribution in vacuum medium associated with moving of electron [26], possible mechanism of spin generation [30] or distribution of electric field around the extended electron [31].

We consider here a simplified model of electron. However its behavior is based on assumptions related to vacuum medium [28]. We discuss this model in order to illustrate role of the chain state. We assume now that electron is a classical point-like particle described by equations

$$
\begin{aligned}
& \frac{\mathrm{d} \boldsymbol{R}}{\mathrm{d} t}=\boldsymbol{v}, \\
& m \frac{\mathrm{d} \boldsymbol{v}}{\mathrm{d} t}=\boldsymbol{f},
\end{aligned}
$$

where $\boldsymbol{R}, \boldsymbol{v}$, and $m$ are position, velocity, and mass of electron, correspondingly.

The force $\boldsymbol{f}$ acting on the electron has more complex form. We assume that this force has two main components

$$
\boldsymbol{f}=\boldsymbol{f}_{u}+\boldsymbol{f}_{a b} .
$$

The component $\boldsymbol{f}_{u}$ represents force induced by electrostatic field only. We do not discuss here any other possible sources of force such as magnetic or gravitational field for instance at this moment.

The component $\boldsymbol{f}_{a b}$ represents influence of nonequilibrium distribution of components of the vacuum medium induced by motion of particle expressed as force acting on the classical point-like particle. Thereby, role of the wave function will be represented just by the force $\boldsymbol{f}_{a b}$.

The force $\boldsymbol{f}_{u}$ is defined in classical way. The force $\boldsymbol{f}_{a b}$ needs a kind of constitutive equations in order to reflect possibility of modelling of a discrete set of electronic orbits or various interparticle interactions.

Motion of the electron is associated with an excess of the component $b$ in front of the particle and a deficiency of this component and also excess of $a$ within zone at the back of the electron. Way of relaxation of this nonequilibrium distribution of components is associated with a system of forces acting on the electron. We assume that

$$
\boldsymbol{f}_{a b}=\boldsymbol{f}_{D S}+\boldsymbol{f}_{b}+\boldsymbol{f}_{a} .
$$

The force $\boldsymbol{f}_{b}$ represents a barrier against motion of the electron due to an excess of the component $b$ in front of the particle and has direction opposite to the motion approximately. The force $\boldsymbol{f}_{a}$ associated with nonbalanced component $a$ behind of the particle, also acts against motion and has direction tangent to the path of the particle. The force $\boldsymbol{f}_{D S}$ is parallel by assumption to the force $\boldsymbol{f}_{b}$ but has the sense the same as velocity and represents dynamics of separation of components in front of the particle.

We assume that the force $\boldsymbol{f}_{a b}$ can be expressed in the form

$$
\boldsymbol{f}_{a b}=f_{D S}(\boldsymbol{v}) \boldsymbol{t}-f_{b}(b) \boldsymbol{t}-f_{a}(a) \boldsymbol{t}_{p t h},
$$

where $\boldsymbol{t}$ is a unit vector which should be determined, $\boldsymbol{t}_{\text {pth }}=\boldsymbol{v} /|\boldsymbol{v}|$ is unit vector tangent to the path of the electron. Functions $f_{b}$ and $f_{a}$ are increasing with respect to $b$ and $a$ by assumption.

The relation (44) indicates that we have to do with variables $a$ and $b$ which are not present in (40) and (41) and should be determined by additional equations. We postulate them in the following forms:

$$
\begin{aligned}
& \frac{\mathrm{d} a}{\mathrm{~d} t}=\bar{c}_{a}(\boldsymbol{v})+c_{J a} \\
& \text { and } \\
& \frac{\mathrm{d} b}{\mathrm{~d} t}=\bar{c}_{b}(\boldsymbol{v})+c_{J b},
\end{aligned}
$$

where $\bar{c}_{a}(\boldsymbol{v})$ and $\bar{c}_{b}(\boldsymbol{v})$ are production of $a$ and $b$ correspondingly owing to velocity of motion $\boldsymbol{v} . c_{J a}$ and $c_{J b}$ stand for production of components due to other mechanisms including divergence of efflux from regions near the electron.

Uniform motion of the electron takes place when $\boldsymbol{f}_{u}=$ $\mathbf{0}$ and also $\boldsymbol{f}_{a b}=\mathbf{0}$. Then, all components of the force $\boldsymbol{f}_{a b}$ are related to a stationary evolution of components where the component $b$ is transferred into back region of particle in order to recover the equilibrium vacuum medium with $\varrho=\varrho_{0}$. Then, we have the relation for uniform motion with velocity $\boldsymbol{v}$ :

$$
f_{D S}(\boldsymbol{v}) \boldsymbol{t}_{p t h}-f_{b}(\bar{b}(\boldsymbol{v})) \boldsymbol{t}_{p t h}-f_{a}(\bar{a}(\boldsymbol{v})) \boldsymbol{t}_{p t h}=\mathbf{0},
$$
where $\boldsymbol{t}=\boldsymbol{t}_{p t h}$.

We see that motion of electron is continued by decomposition of vacuum medium represented by $\varrho$ into components $a$ and $b$. As a result of this $a$ goes into electron and $b$ appears in excessive form before the particle in region denoted by $B_{F}$. Waving associated with electron motion results from relaxation of the component $b$ towards $a$ stopped behind the electron in region denoted here by $A_{B}$, in order to recover equilibrium vacuum medium $\varrho$. Character of waving in this case is described in [26].

Let us consider the situation when another electron moves in similar direction before the first one. Then, in front of the first electron a region $A_{B 2}$ appears. It induces transfer of the component $b$ from the region $B_{F 1}$ directly to the $A_{B 2}$. Neglecting at this moment electrostatic repulsion we see that the two electrons interact by the forces in the vacuum medium. We say that the two particles are in an open chain state. 
We can have also to do with closed chain when the set of particles, one behind another, creates a ring when the last particle is behind the first one and all particles transfer the component $b$ from the region $B_{F k}$ to the region $A_{B(k+1)}$. Stationary orbit of electron can also be interpreted as closed one-particle chain state. This is so since the way of transferring of the component $b$ is not interpreted as transfer onto back of the particle but into front of the particle.

Let us discuss an open chain of two electrons. Then, transfer of the component $b$ towards $A_{B 2}$ induces decrease of $b$ in $B_{F 1}$. However, at the same moment this component is not transferred into $A_{B 1}$ and $a$ is not efficiently reduced to $\varrho$. Thereby we have to do with the following situation:

$$
\begin{aligned}
& a \nearrow a \in A_{F 1}, \quad b \searrow b \in B_{F 1}, \\
& a \searrow a \in A_{F 2}, \quad b \nearrow b \in B_{F 2} .
\end{aligned}
$$

This in turn means that

$$
f_{a 1} \nearrow, f_{b 1} \searrow, \quad f_{a 2} \searrow, f_{b 2} \nearrow .
$$

This behavior of forces follows that the particles attract themselves. In this interpretation we have to do with hidden assumption that lowering of $f_{b 1}$ is more effective for acceleration of the first particle than lowering of the force $f_{a 2}$ for acceleration of the second particle.

On the other hand, whole chain system is slowing down since the forces $f_{a 1}$ and $f_{b 2}$ have increased. In other words whole system have more difficult way of transferring of the component $b$ from $B_{F 2}$ to $A_{B 1}$ which is due to larger distance between the two regions than in case of the free particle.

The situation qualitatively changes when the last particle of the $n$-particle chain state can go directly behind of the first one. Then transfer of the component $b$ is more easy. We postulate the following hypothesis for closed chain of particles:

$$
\left[\boldsymbol{f}_{D S}-\boldsymbol{f}_{b}-\boldsymbol{f}_{a}\right]_{k} \boldsymbol{t}_{p \operatorname{th}(k)}>0
$$

for each $k$-th particle of the chain state in an idealized moment where particles are distributed uniformly on a circle and there is a possibility for decrease of $D_{a b}$, where $D_{a b}$ represents quantity similar to length of wave considered between neighbouring particles, see $[26,28]$ for more details. This assumption means that the closed chain of particles with the same charge accelerates when we neglect other forces acting on particles and furthermore the circle undergoes a shrinking in order to maintain properties of the relation $D_{a b}=D_{a b}(v)$. This means also that corresponding chain state is associated with a centripetal force induced among others by space distribution of components during this motion.

Such a perfect circular chain is disturbed in reality by some forces and is not uniform in discussed above sense. However, by this assumption we indicate a general property related to dynamics. In particular, one-particle chain state considered as electron on its orbit around a nuclei, is uniform in discussed above sense and will accelerate in absence of other forces.
Properties of the chain state of particles discussed above suggest directly that such a state could happen in molecular systems in closed non-circular form or as open chain. Consequently, corresponding chain state of electrons in molecules interact with nuclei by tendency to its shrinking. Then, opposite charge of nuclei acts against this process. However, tendency to shrinking can be interpreted just as action of an attractor which governs this nuclei system.

Let us note that chain state of electrons in molecules should be interpreted as an excited state. This is so since distances between electrons in chain states are smaller than lengths of electron orbits. The relation $D_{a b}=D_{a b}(v)$ [28], which is decreasing function representing length of wave associated with electron, indicates that velocities of electrons in chain state are then larger than in orbital motion. Attractor in molecular system should contain electronic attractor as its main part. In general in molecules we have to do with conjugation of electronic motion and nuclei motion. Thereby description of whole attractor needs full description of nuclei, their wave function and characterization of interactions between nuclei and electrons also by their wave functions interpreted as nonequilibrium distribution of vacuum medium components. Full description of this kind is expected in a future.

\section{Stabilization of vacuum medium mechanics as fundamental theory}

\subsection{Introduction}

Within vacuum medium mechanics we have obtained considerable number of results which supports our aims leading to determination of source of precision for nanotechnological processes. We try also to improve discussed previously unified mechanics of materials towards extending it to condensed matter theoretical physics. We state here that vacuum medium mechanics can be incorporated into multiscale method of modelling called here collection of dynamical systems with dimensional reduction. Thereby, it seems possible that various aspects of condensed matter theoretical physics could cooperate within one theoretical scheme with mechanics of materials. As a result of this vacuum medium mechanics is viewed also as a tool supporting design of nanotechnological devices.

Consequently we state that vacuum medium mechanics could be appropriate basis for our unified description. It means that future development could be based on this theory. Fundamental theory considered as appropriate basis for our unified description should be stable with respect to correctness of description of reality. Therefore such a stability will be discussed in what follows. We should estimate to what degree vacuum medium mechanics describes reality correctly taking into account its relation to experimental observations as well as to methodology of description of reality. In other words we should estimate to what degree we can stand on the vacuum 
medium mechanics as fundamental theory for further development of theoretical descriptions leading to theoretical nanotechnology.

The most important is to investigate status phenomenon of the chain state of particles with the same charge as of key importance for determination of attractor responsible for self-organization. However, vacuum medium mechanics is seen as fundamental theory. Therefore it should describe efficiently all important observed phenomena in all scales including astrophysical processes. In case when vacuum medium mechanics would be in contradiction with astrophysical phenomena for instance we would presume that its role as fundamental theory is not appropriate.

Consequently we discuss status of vacuum medium mechanics with respect to elementary particle physics experiments, with respect to astrophysical observations and also with respect to its ability to description of biological processes. Importance of the last aspect follows that consequences of vacuum medium mechanics for modelling of biological processes are discussed in separate, next section. We estimate in this section status of the chain state of particles with the same charge with respect to methodology of modelling as well as with respect to experimental observations.

Accordance of vacuum medium mechanics with large set of experimental results would provide us conviction that this is appropriate basis for development of engineering in small scales.

\subsection{Status of vacuum medium mechanics with respect to experimental observations related to various elementary particle interactions}

Vacuum medium mechanics is introduced by means of entirely new system of fundamental notions. It means that this theory is not obtained by modification of existing ones. New system of assumptions is constructed in order to describe well known results from experimental physics and also for introduction of new quality especially in description of dynamics on the most fundamental level. Methodology applied to construction of vacuum medium mechanics is elaborated in order to provide possibility of estimation of status and correctness of introduced assumptions [32].

Description of vacuum medium was constructed taking into account well known properties of elementary particles. Therefore within vacuum medium mechanics such properties are described. Let us mention attraction of particles with opposite charge, gravitational attraction of massive particles, electric and magnetic properties of electron for instance.

However, we obtain also entirely new properties of elementary particles. Our particles are not point-like. They are extended objects. Just owing to this property we are able to describe mechanism of breaking of charge conservation law. This leads to considering proton as composed of three-positrons in the chain state. Status of the chain state will be discussed further where we will discuss also status of concept of three-positron proton.
Within vacuum medium mechanics neutrino can create bounded unstable state with charged particle. This is important prediction which creates new view on internal structures of unstable elementary particles [35]. Let us mention neutron for instance which is seen now as bounded state of proton, electron, and antineutrino which is in accordance with its decay.

Let us note that recently discovered boson $\mathrm{E}(38)[33,34]$ is interpreted by vacuum medium mechanics as bounded state of one electron and one neutrino [35]. This particle does not fit to the standard model and is not predicted within it. Therefore this discovery considerably increases status of vacuum medium mechanics. Summarizing we obtain the following remark:

Remark 5.1: System of fundamental notions related directly to vacuum medium is placed below elementary particle level. As a result of this we can describe particles as extended objects within vacuum medium and having its own internal dynamics. This increases considerably resolution of seeing of reality by theory. It leads directly to prediction of existence of bounded unstable state of electron and neutrino with mass between $0.5 \mathrm{MeV}$ and $105 \mathrm{MeV}$. Therefore discovery of boson E(38) interpreted just as particle having mentioned above properties, and which is not predicted by other current fundamental elementary particle theories, considerably supports vacuum medium mechanics.

It is also important to note that observed lately neutron halo where neutron goes around nuclei with distance exceeding range of strong interactions suggests importance of the chain state. Let us note that neutron is composed of proton, electron, and neutrino in bounded unstable state within vacuum medium mechanics.

In the case of neutron halo neutron can be considered to be in one particle closed chain state where components are transferred into back of neutron along its orbit instead of transferring them around of neutron as this is the case in its free motion. In accordance with our previous discussion on chain states we have to do in this case with centripetal force which does not happen in quantum mechanical description. It means that circular orbit of neutron is then possible. This discovery also considerably increases status of vacuum medium mechanics.

\subsection{Status of vacuum medium mechanics with respect to astrophysical observations}

Consequences of vacuum medium mechanics for astrophysics have been investigated in [27]. In this paper one defines black hole state of particles.

Several properties of model of vacuum medium have important consequences. The first one states that elementary particles can leave black holes in case when they have sufficiently high energy. This is not the case for electromagnetic waves. Difference follows from fact that mechanisms of motion of particle and propagation of electromagnetic wave are different. The second consequence is associated with explosion of space during annihilation of electron and positron. Both particles are 
not point like. Therefore joining of electron and positron leads to emission of energy by electromagnetic waves and also changing of volume of corresponding joined components in comparison with separated ones.

The last property leads to Big Bang model based on annihilation of electrons on protons on the inside of giant black hole. As a result of annihilation of large number of electrons on positrons from proton giant explosion of space happens. This explosion is not uniform and part of matter remains. We observe this matter today as astronomical objects. After this explosion we have to do with internal black hole wall which surrounds our space.

Consequently, our observed Universe is placed on the inside of black hole. All galaxies fall into internal black hole wall. This explains directly why galaxies accelerate during expansion of observed Universe. This explains directly problem of dark energy which is interpreted as associated with gravitation of external part of the black hole which is not destroyed by explosion of space.

Problem of symmetry of matter and antimatter is solved directly. It is associated with initial conditions for explosion of space. In these conditions we have not to do with symmetrical amount of matter and antimatter. Therefore this is the case also for further evolution of Universe.

Explosion of space should be fading. As a result of this larger amount of matter is placed nearby to internal black hole wall in comparison with center of space. This explains large amount of quasars and supermassive black holes far from us. This fact is in contradiction with traditional Big Bang model where far from us we should observe light objects.

We should accentuate that observed fact of existing of considerable amount of positrons around supermassive black hole in center of our galaxy can be interpreted as resulting from smaller explosions of space on the inside of this black hole. Corresponding explosions could be induced by energy provided by accretion disc. As a result of annihilation of electrons on protons two excessive positrons are produced. Positrons could leave black hole, in accordance with vacuum medium mechanics, and should be present in relativistic jets produced by the black hole.

Summarizing, astrophysics provides at this moment the most convincing arguments for validity of vacuum medium mechanics. Therefore just such consequences have been investigated in the paper [27] and are discussed here.

\subsection{Status of notion of the chain state with respect to experimental observations and methodology of modelling of reality}

Previous discussion shows that the chain state of particles determines attractor responsible for self-organization in molecular systems. Thereby we should estimate status of this notion in theoretical physics. We expect high status which means that we should have conviction that such a chain state indeed happens in reality and can play role assigned to it.
Let us discuss first methodological aspects related to this notion. In the paper [36] we discuss how logic could happen in evolving dynamical systems. In particular implication happens as a result of action of main dynamical system [36] which leads to physical interpretation of mathematical logic [36]. Furthermore we obtain by these considerations some methodological premises for the question how to construct fundamental notions for physical theory describing reality $[32,36]$.

Cognition realized by means of action of a dynamical system [36] indicates that we recognize an external world approximately only. This in turn means that the term "true" is not adequate to characterize such a situation. The term "true" is appropriate in internal deduction of the brain where we determine kind of logic, methods of proof and so on and as a result of this we are on the inside of a mathematical system.

In case of theories describing a reality we believe that corresponding theories describe well this reality. Therefore, the term "status" of the theory would be better for characterizing quality of approximation of reality. In this case the term "true" appropriate for mathematical theorems is too radical in case of description of reality associated with external world not entirely recognized and seen only by mathematical theory.

Consequently high status of theory means that we believe that the description of reality by corresponding theory is good in some sense. Consequently it is important for us to estimate which theories have a high status and which have the low one.

First, let us notice that set of terms of theory [36] is interpreted as the simplest sensorial information. This provides us premises for methodology of construction of set of fundamental notions for theory describing reality. Fundamental notions should be related to the simplest information following from sensors. However, what is it the simplest information.

One suggests to interpret the simplest information as that one which is associated with maximum engagement of the internal neural network system for interpretation of the sensory results [36] and this interpretation is manifested by relatively simple formulae within the neural network.

Remark 5.2: Fundamental notions of theory describing reality should be related to maximum engagement by action of the dynamical system and its sensory system in order to obtain results which next can be interpreted as the simplest information by neural network system. Then, status of such a fundamental notion is viewed as high.

Let us consider an example. We observe creation of electron-positron pair. Within our current experimental possibilities, using maximum efforts we are able to state that some components are separated and we call these components by $a$ and $b$ correspondingly by the simplest formulae without any understanding of the component. Consequently, the fundamental notions are then expressed as follows: the component $a$ exists and the 
component $b$ exists without more particular specification of properties of these components. Such fundamental assumptions have high status in description of reality by theory constructed on such assumptions.

Assumptions can appear in theory as a result of a mathematical proof following from some external considerations. Then, the larger length of this proof the lower status of fundamental assumption considered. In other words larger length of this proof is interpreted as associated with higher resolution of the brain applied for interpretation of various steps. Then probability of correct approximation of reality decreases.

In order to increase status of fundamental assumption based on longer proof we should have at our disposal several proofs. They should start from several independent points and lead to the same consequence which is next considered as a fundamental assumption.

Consequently we have discussed above that status of existence of components $a$ and $b$ is high. It means also that status of description of motion of electron composed of $a$ component is high. Indeed motion has to be continued by separation of component $a$ and $b$ from equilibrium $\varrho$. Then $a$ goes into electron and $b$ has to relax and finally join with component $a$ behind of electron where such a component is stopped, in order to recover equilibrium vacuum medium $\varrho$. This is simple and direct consequence of existence of $a$ and $b$ which confirms high status of this qualitative description of motion of electron.

Chain state of electrons, in accordance with previous considerations, is also direct and simple consequence. This is so since recovering of equilibrium vacuum medium can happen also by joining of excessive $b$ in front of one electron with stopping component $a$ of another electron. Summarizing we have to do in all discussed above cases with simple and direct inferring from high status assumptions on existence of of components $a$ and $b$ which means that status of notion of the chain state is high.

The question is where we can find experimental confirmation for existence of the chain state. Within vacuum medium mechanics proton is composed of three positrons in the chain state. In the paper [29, 35] we discuss experimental evidence for this fact. We have found there eleven independent arguments for three-positron structure of proton as follows:

1. The same external effect for positron and for proton is represented by their external electrostatic field which indicates that both particles are composed of the same component.

2. Generation of neutrino under high compression approaching electron and proton suggests that we have to do with both $a$ and $b$ components necessary for production of rotating $\varrho$ interpreted just as neutrino.

3. Annihilation of proton and antiproton predominantly to three electrons and three positrons.

4. Annihilation of proton and antiproton through intermediate particle-antiparticle pairs instead of direct annihilation to electromagnetic waves. This means that direct production of electromagnetic waves is difficult since three electron-positron pairs have to annihilate almost simultaneously which exceeds critical conditions for production of single electron positron pair. As a result of this larger non-stability of vacuum medium has to produce additional particles instead of pure electromagnetic waves. This effect is expected just for three-positron proton.

5. Presence of positrons near supermassive black hole in center of our galaxy resulting from annihilations of electrons on protons on the inside of black hole [27].

6. Presence of positrons in quasar jets resulting from annihilations of electrons on protons on the inside of black hole [27].

7. Consistency of the Bing Bang theory based on annihilation processes with astrophysical observations [27].

8. Possible explanation of gamma ray bursts as collisional processes of black holes.

9. Interpretation of the three-positron structure of proton as three-particle chain state which can be considered as an asymptotic state for an attractor. Difficulties with disintegration of proton suggests that the attractor represents considerable power. It provides arguments for mechanisms of long lasting evolution and their persistence taking into account chain states of electrons in molecular systems.

10. Decay of $J / \psi$ meson into proton-antiproton pair, resulting from conditions leading to creation of corresponding chain states of three positrons and three electrons within the neutral meson.

11. Relatively long lifetime for the meson $J / \psi$ perhaps induced by pre-proton and pre-antiproton chain states.

Above arguments represent various types of experimental results and astrophysical observations. It makes almost sure that the proton is indeed composed of three positrons in chain state.

Summarizing the whole above discussion we can state that notion of the chain state of particles with the same charge has high status confirmed by methodology of description of physical reality as well as by large spectrum of experimental results.

\section{Relations between theoretical biology and engineering in context of theoretical nanotechnology}

\subsection{Necessity of consistent development}

of theoretical biology and small scale engineering

We observe nanotechnological processes in cell. This means that within our unified description for small scale engineering biological processes should also be described. This means also that mechanisms of biological evolution and definitions of life should be expressible within approach with vacuum medium mechanics.

Let us also note that living organisms determine aims for various scale engineering. This induces directly necessity of consistent description of biology and nanotechnology. In particular we can expect that small scale engineering will cooperate with medicine where human organism would determine character of corresponding engineering. 
Discussion on theoretical biology based on vacuum medium mechanics carried out here is seen also as element of stabilization of vacuum medium mechanics as fundamental theory.

\subsection{Towards definition of life}

We do not encounter in literature any attempt to understanding mechanisms of evolution based on the most fundamental level of description related to elementary particles. As a result of this also life as such is not appropriately defined.

We encounter in literature various attempts to defining of life. Let us mention [37-39] for instance. Following these papers we come to the conclusion that we have not at our disposal satisfactory definition of life. In particular it is difficult to discriminate living systems and not living systems.

Life is frequently defined as system of chemical reactions. Such a property is rather not appropriate for characterizing living systems in general. We have to do in living molecular systems with subsystems which are stable and are rather similar to solids. Frequently they are catalysts of chemical reaction. Even processes related to DNA are rather not chemical reactions. They are characterized by considerable conformational changes. Let us mention unwinding of DNA. This is not chemical reaction. This is separate process which can be accompanied by chemical reactions. Replication of DNA is associated with large number of processes which are not interpreted directly as chemical reactions.

We would say that living organisms are systems which are open with respect to matter and energy and are able to evolve. Just this ability to evolution in short and longer time needs fluent transformation of structures. Corresponding transformation of structures means just that chemical reactions have to take part in this. Thereby, chemical reactions are rather manifestation of possibility of transformation of structures than they are representing the essence of living systems.

Previous discussion on attractor governing selforganization could suggest very extended definition of life as related to each chain state which apears in molecular systems. However, such a definition seems to be too simplified. An autonomy and possibility of evolution seems to be more important feature of life. In the context of interpretation of the chain state of particles we would say that situation when the chain states try to evolve and defense against their destruction by subordination of other molecular processes better fits to life definition. Let us also mention that electronic chain state considered within molecular systems is seen as excited state. This means that we have to do in this case with an open energetically system. Consequently I propose the following definition:

Definition 6.1: Molecular system open energetically and open with respect to mass integrated by various interactions induced by chain states for maintaining existence and evolution of these states is defined here as life.

We see that no replication is discussed here. Indeed we should accept the situation when evolution of the chain state in open system does not encounter molecules leading to division of this system. Thereby such a kind of life can exist and fade in an environment.

We have applied in above definition the term "integrated by various interactions". Precise definition of this term is to some degree open question. Various definitions of integrity property for reacting molecular systems are discussed in [40-42]. This term should be redefined gradually with development of vacuum medium mechanics and corresponding molecular theoretical physics. Let us note that concept of energy barrier zone of activity [28] associated with the chain state provides a context for discussion of increased activity of molecular systems around the chain state when the chain state defends its existence. Then, methods of modelling of feeding of the system could be emerged.

Let us consider evolution of a molecular system towards proteins and perhaps some RNA neglecting at this moment how it could happen. We can admit that RNA can be partially replicated during this stage of evolution. This replication can be highly asymmetric. Then we encounter a proliferation in general. Such a life could fade in energetically rich environment and next could be generated again in changing forms.

Definition 6.2: Let us consider molecular system open energetically and open with respect to mass integrated by various interactions induced by chain states for maintaining existence and evolution of these states. Let us assume that transfer of energy towards this molecular system leads to division of this system, including separation of integrated chain states. As a result of this two other systems with similar properties as initial system are created. Then such a system is called the life with proliferation.

In this case we admit the situation where division of molecular system with the chain states happens. The division can result from appearing of molecule undergoing division in this system. This division could be rather arbitrary and not necessarily similar to replication. Such a kind of life could appear when protein-RNA system attains the stage when RNA could be partially replicated and create separate protein-RNA system. Such a situation will be discussed in what follows. This process is not treated as replication. Therefore the term proliferation is applied. In the case of replication we have to do with division into two similar objects with small differences with respect to initial object. Consequently we can introduce also the following definition:

Definition 6.3: Let us consider molecular system open energetically and open with respect to mass integrated by various interactions induced by chain states for maintaining existence and evolution of these states. Let us assume that transfer of energy towards this molecular system leads to division of this system, including separation of integrated chain states. As a result of this two other systems with similar properties as initial system and with structures which are in both cases similar to 
initial system are created. Then such a system is called the life with replication.

We have introduced definitions of life which are based on the notion of chain state of particles with the same charge in molecular system. However we should be careful. This is so since with development of vacuum medium mechanics we can encounter necessity for extension of system of fundamental notions and take into considerations some perhaps more fundamental and subtle properties of the vacuum medium which take part in selforganization processes. Nevertheless at this moment we have at our disposal definition of life which is based on attractor considered on the most fundamental level of theoretical description.

\subsection{Discussion of mechanisms of biological evolution based on vacuum medium mechanics}

We would like to elucidate role of vacuum medium mechanics for theoretical biology by expression of important concepts for this field. At this moment we have not at our disposal fully described molecular physics based on the vacuum medium mechanics. However, some properties of electron theory hitherto obtained are useful for prediction of various phenomena in molecular systems [26, 28, 30, 31]. In Ref. [28] mechanism of bonding of two hydrogen atoms is discussed. This is an initial step for constructing theoretical molecular physics and theoretical chemistry on basis of the vacuum medium mechanics.

Having at our disposal mentioned properties of electronic systems we will try to express mechanism of biological evolution by set of hypotheses and qualitative thinking. All this should be useful as conceptual framework for development of molecular physics based on vacuum medium mechanics.

We would like to characterize how chain states of electrons could act as self-organization mechanism within molecular systems. To this end we introduce set of spaces where the problem will be expressed.

Let us consider configuration space $\mathcal{V}_{C}$ which contains vectors $\boldsymbol{R}=\left\{\boldsymbol{R}_{1}, \ldots, \boldsymbol{R}_{N}\right\}$ representing position of atomic nuclei in a molecular system. Then, $\mathcal{V}_{D}$ is corresponding phase space.

We accentuate role of electronic structure and its dynamics in self-organization process. Thereby we should characterize evolution of electronic structure. Therefore we introduce also corresponding configuration space $\mathcal{V}_{C e}$ which contains position vectors for electrons $\boldsymbol{r}_{e}=$ $\left\{\boldsymbol{r}_{1}, \ldots, \boldsymbol{r}_{M}\right\}$ and corresponding phase space $\mathcal{V}_{D e}$ for our electronic system. All this does not mean that we are able to measure corresponding electronic positions. However, within our point-like approximation discussed previously such positions can be expressed within mathematical description.

Consequently we have spaces for description of nuclei and electronic dynamics. However, this dynamics is governed by multicomponent wave function composed of values $a, b, \varrho$ in each point of space. Conequently, we introduce also configuration space $\mathcal{V}_{\text {Cab }}$ of all admissible functions $\{a, b, \varrho\}(X)$ and corresponding phase space $\mathcal{V}_{\text {Dabe}}$.

Summarizing our problem will be described by a dynamical system with variables within the following space:

$$
\mathcal{V}=\mathcal{V}_{D} \times \mathcal{V}_{\text {De }} \times \mathcal{V}_{\text {Dabe }} \times \mathcal{V}_{\text {Dext }}
$$

where we consider additionally possibility of external interactions by presence of external molecules represented by presence of corresponding phase space $\mathcal{V}_{\text {Dext }}$.

We are not able at this moment to express full equations of evolution of molecular system within vacuum medium mechanics, which happen in the space (51). However, we are able to carry out some simplifications and approximations in order to express our ideas taking into account quality of description provided by current state of vacuum medium mechanics. In particular various projections of solutions between spaces are possible in order to provide environment for interpretation of various phenomena in qualitative way.

Let us introduce set of notions which will allow us to express concept of driven force for biological evolution.

We will use potential energy $V(\boldsymbol{R})$ especially for characterization of evolution of nuclei of our molecular system which can undergo chemical reactions. Consequently, we introduce catchment regions $[43,44]$ where minima of the potential energy are present.

Let $C_{P}$ be the catchment region related to minimum $\boldsymbol{R}_{m} \in C_{P}$. All steepest descent paths $[43,44]$ with origin in $C_{P}$ tend to minimum $\boldsymbol{R}_{m}$. We will discuss also index of minima. The saddle point $\boldsymbol{R}_{s p}$ is considered as a critical point with index 1 (one negative eigenvalue of the corresponding Hessian) [43, 44]. The minimum $\boldsymbol{R}_{m}$ in $C_{P}$ is considered as critical points with index 0 .

We can discuss path of chemical reaction as the steepest descent path $p\left(\boldsymbol{R}_{s p}, s\right), s \in\left[s_{1}, s_{2}\right]$ with the origin at the point $\boldsymbol{R}_{s p}$ and the end at critical point $\boldsymbol{c}=\boldsymbol{R}_{m}$. However, we know that in reality thermal processes induce real reaction path as lying in a neighbourhood of the steepest descent path. In order to characterize such a situation we introduce here concept of dynamical path.

We project dynamics into configuration space. Idea of this projection consists in introducing a set of possible trajectories accompanied by the steepest descent path with the origin $\boldsymbol{R}_{s p}$ as a saddle point. The projection is referred also to the set of initial conditions $D_{I C}$. Let us introduce catchment regions $C(0, i)$ with number $i$ and with corresponding minimum of index 0 , a saddle point $\boldsymbol{R}_{s p} \in \partial C(0, i)$ and the steepest descent path $p\left(\boldsymbol{R}_{s p}, s\right)$. We assume that $\boldsymbol{R}_{s p}=\boldsymbol{c}(1, i) \in C(1, i) \subset \partial C(0, i)$ for some value of $i$.

Let $\pi_{C}: \mathcal{V}_{D} \rightarrow \mathcal{V}_{C}$ be the projection of the phase space on the configuration space. Let $U_{I C}=\pi_{C}\left(D_{I C}\right)$ and $U_{I C} \subset C(1, i), \boldsymbol{R}_{s p} \in U_{I C}$. By this projection we understand the representation of all trajectories determined in phase space, by trajectories in configuration space corresponding to a set of initial conditions, where dynamics is not observed. However dynamics is then manifested by various deviations of trajectory from steepest descent 
path. We assign a set $U\left(p, D_{I C}\right)$ to $p$. This set represents possible trajectories with initial conditions belonging to the set $D_{I C}$.

Let $D_{I C}$ stands for the set of initial conditions which corresponds to all states which are possible for description of our process of chemical reaction. We introduce the following definition.

Definition 6.4: The dynamical path is a set $U\left(p, D_{I C}\right) \subset C(0, i) \subset \mathcal{V}_{C}$ representing trajectories obtained by means of $\pi_{C}$ for dynamical processes with initial conditions $D_{I C}$ and assigned to the steepest descent path $p\left(\boldsymbol{R}_{s p}, s\right)$ with property that $U\left(p, D_{I C}\right) \cap \partial C(0, i)=$ $U_{I C}$ and $\left\{\boldsymbol{R}_{0}, \boldsymbol{v}_{0}\right\} \in D_{I C}$.

Connection of the dynamical path with the steepest descent path rests on option of initial conditions $U_{I C}$ as lying on $\partial C(0, i)$. The catchment regions of $C(0, i)$ type will be denoted by $C_{\alpha}$ and minimum corresponding to them by $\boldsymbol{c}_{\alpha}$.

Let us consider two adjacent catchment regions $C_{\alpha}$ and $C_{\beta}$ and their topological closures $\bar{C}_{\alpha}, \bar{C}_{\beta}$. We define the set $U_{\alpha \beta}\left(p_{\alpha}\right)=U_{\alpha}\left(p_{\alpha}, D_{I C}\right) \cap \bar{C}_{\alpha} \cap \bar{C}_{\beta}$. We can discuss a process with a jump between various catchment regions. Such a jump is possible if the dynamical path attains a part of boundary of $C_{\alpha}$. This is characterized by the set $U_{\alpha \beta}\left(p_{\alpha}\right)$. If $U_{\alpha \beta} \neq 0$ then the jump from $C_{\alpha}$ to $C_{\beta}$ is possible.

If $U_{\alpha \beta}\left(p_{\alpha}\right) \cap C\left(1, \boldsymbol{R}_{s p \beta}(i)\right) \neq 0$ and $U_{\alpha \beta}\left(p_{\alpha}\right) \cap$ $C\left(1, \boldsymbol{R}_{s p \beta}(j)\right) \neq 0, i \neq j$, then two possible steepest descent paths can be considered in $C_{\beta}$.

It is also possible that $U_{\alpha \beta}\left(p_{\alpha}\right) \cap C\left(1, \boldsymbol{R}_{s p \beta}(i)\right) \neq 0$ and $U_{\alpha \gamma}\left(p_{\alpha}\right) \cap C\left(1, \boldsymbol{R}_{s p \gamma}(j)\right) \neq 0$. Then, transition from $C_{\alpha}$ to two other catchment regions, $C_{\beta}$ and $C_{\gamma}$, is possible.

Definition 6.5: We say that two dynamical paths $U_{\alpha}\left(p_{\alpha}\right)$ and $U_{\beta}\left(p_{\beta}\right)$ are $(\alpha, \beta)$-successive if $U_{I C \beta}=$ $U_{\alpha \beta}\left(p_{\alpha}\right) \cap C\left(1, \boldsymbol{R}_{s p \beta}\right) \neq \emptyset, \boldsymbol{R}_{s p \beta}$ is the origin of $p_{\beta}$, $\pi_{C}\left(D_{I C \beta}\right)=U_{I C \beta}$ and $U_{\beta}\left(p_{\beta}\right)$ is assigned to $p_{\beta}$.

Definition 6.6: We say that the set of dynamical paths $\left\{U_{\alpha}\left(p_{\alpha}\right)\right\}, \alpha \in I_{A}$ is the descent chain of dynamical paths if for each $\alpha=1, \ldots, A-1, U_{\alpha}\left(p_{\alpha}\right)$ and $U_{\alpha+1}\left(p_{\alpha+1}\right)$ are $(\alpha, \alpha+1)$-successive and $V\left(\boldsymbol{c}_{\alpha}\right)>V\left(\boldsymbol{c}_{\alpha+1}\right)$.

Let $\boldsymbol{c}_{A}, \boldsymbol{c}_{B}$ be two minima of the potential energy associated with a dynamical process.

Definition 6.7: We say that $\boldsymbol{c}_{A}, \boldsymbol{c}_{B}$ are joined by dynamical processes if there exists a descent chain of dynamical paths with an initial path $p_{i}$ with the end $\boldsymbol{c}_{A}$ and a final path $p_{f}$ with the end $\boldsymbol{c}_{B}$. We denote such a dynamical path by $U\left(\boldsymbol{c}_{A}, \boldsymbol{c}_{B}\right)=\bigcup_{\alpha} U_{\alpha}$.

Now we can consider a reaction which is continued through a set of critical points with a shallow valley. Activity of such a process follows from dynamics provided by temperature. Let us note that the dynamical path can have several ends corresponding to various critical points. Indeed, when some regions of potential energy hypersurface are flat and have several minima or are small then the dynamical path can go in various directions. Such a situation is possible just in case of complex molecules.
Then, forking of the dynamical paths happens not only on saddle point but also in discussed above regions.

Above introduced notions can be helpful for characterization of evolution of molecular system when we apply traditional description with well defined potential energy hypersurface following from electronic processes. However, we expect a new quality when we will consider additionally chain states of electrons in case when they are extended on larger distances within molecular system.

Let us consider a chain state of electrons in its stationary state. We assume then that electrons are moving with constant averaged velocity $\left|\boldsymbol{v}_{C H S}\right|$ and constant averaged distance between them $d_{C H S}$. We assume furthermore that distance between electrons is connected with velocity by relation $\left|\boldsymbol{v}_{C H S}\right|=D\left(d_{C H S}\right)$ which is represented by positive and decreasing function having similar role as length of wave assigned to particle [28].

We can also introduce concept of potential energy related to distance between electrons corresponding to this dynamical case. The potential energy reflects the fact that given distance between electrons is associated with a distribution of components $a, b, \varrho$.

We assume that we are able to approximate influence of electronic structure on nuclei motion by a potential energy $V$. We assume additionally that we are able to represent such a potential energy as a sum of components

$$
V=V_{M}+\sum_{i} V_{C S i}, V_{C S i}=V_{P i}+V_{C S L i} .
$$

In this expression $V_{M}$ stand for part of potential energy which is not related directly to the chain state. The component $V_{C S i}$ is associated with $i$-th chain state. In particular $V_{P i}$ is responsible for polarization of atom due to transfer of electron from atom to atom by $i$-th chain state. The component $V_{C S L i}$ is responsible for a longitudinal force acting along the chain state related just to the distance between electrons.

Consequently $V_{C S L i}$ depends on distribution of components $a, b, \varrho$. Distribution of components depends in turn on evolution of distribution of these components described by separate equations. We should characterize this dependence as well as role of part of potential energy $V_{C S L i}$ for nuclei motion. It is expected that $V_{C S L i}$ within this approximation could be responsible for generation of narrow valley in region where considerable number of equilibrium configurations for large molecule is possible. Thereby, activation of the chain state should lead to reduction of possible configurations of larger molecules. We identify $V_{N e}$ as follows:

$$
V_{N e}=\sum_{i} V_{C S L i} \text {. }
$$

We assume that $V_{N e}=V_{N e}(\boldsymbol{R}, \boldsymbol{d})$, where $\boldsymbol{d}=$ $\left\{d_{1}, \ldots, d_{C H}\right\}$ represents distances between successive electrons in chain state. Let us note that $V_{N e}$ represents effect of the chain state on nuclei motion. Therefore this function depends on $\boldsymbol{R}$.

We can consider formally potential energy for various configuration spaces $\mathcal{V}_{C}, \mathcal{V}_{C e}$ and $\mathcal{V}_{\text {Cabo }}$. Then these 
functions could characterize motion of nuclei, electrons and corresponding components of the vacuum medium. However, it is difficult to separate them entirely. Therefore hitherto introduced $V_{N e}=V_{N e}(\boldsymbol{R}, \boldsymbol{d})$ could be reinterpreted going towards obtaining also potential energy governing electronic motion. Then pure electronic potential energy $V_{e}$ should depend on $\boldsymbol{r}_{e}$ and also other configurational variables. We introduce formally $V_{e}=$ $V_{e}\left(\boldsymbol{d}, \boldsymbol{r}_{e}, \boldsymbol{R}\right)$. Let us note that $\boldsymbol{d}$ is defined by means of $\boldsymbol{r}_{e}$. However we discriminate these variables since $\boldsymbol{d}$ are chosen for characterization of properties of the chain states. We should take into account also effects which are described by variables from $\mathcal{V}_{\text {Cab } \varrho}$.

Consequently we have $V=V(\boldsymbol{R} ; \boldsymbol{d}, \xi)$ and $V_{e}=$ $V_{e}\left(\boldsymbol{d} ; \boldsymbol{r}_{e}, \boldsymbol{R}, \xi\right)$, where $\xi$ represents variables responsible for evolution of compoenents $a, b$ and $\varrho$ perhaps in some simplified form.

Let us consider the chain state of electrons having determined sets $B_{F k}$ and $A_{B(k+1)}$ as regions where excessive $b$ component in front of $k$-th particle and excessive unbalanced $a$ behind of $k+1$ electron are present. As a result of this transfer of $b$ component happens by a flux $J_{b}$ towards $A_{B(k+1)}$ in order to recover stable vacuum medium $\varrho$.

We would like to characterize possible disturbance of this process when motion of corresponding electrons is continued. To this end we consider distribution of the component $b$ within $B_{F k}$ by approximation with the aid of several values $\left\{b_{k j}\right\}$, corresponding to chosen points of space placed within $B_{F k}$, where $j=1, \ldots N_{k}$. Similarly we consider distribution of the component $a$ in $A_{B(k+1)}$ by discrete values $\left\{a_{(k+1) m}\right\}$ corresponding to chosen points of space placed within $A_{B(k+1)}$, where $m=1, \ldots, N_{k+1}$. By analogy we introduce values of $b_{(k+1) m}$ for approximation of distribution of $b$ in $A_{B(k+1)}$ and $a_{k j}$ for approximation of distribution of $a$ in $B_{F k}$.

Let us note at this place that the wave function is associated with evolution of $\{a, b, \varrho\}$ in each point of space around the particle. It means that various amounts of $a$ and $b$ can be present in regions $B_{F k}$ and $A_{B(k+1)}$. In particular, $\varrho$ can be considered as $\{a, b\}$, where $a=b$ as bounded within $\varrho$ component. We can expect that in some neighborhood of $B_{F k}$ an additional region $A_{E X T}$ where excessive unbalanced $a$ could happen. We approximate corresponding distribution of $a$ by $\left\{a_{E X T p}\right\}$.

Above approximations of distribution of components allow us to discuss tendency where components should flow.

Let us consider quantities associated with regions $B_{F k}$ and $A_{B(k+1)}$ by

and

$$
G_{b j m}=\frac{\left(b_{k j}-b_{\varrho k j}\right)-\left(b_{(k+1) m}-b_{\varrho(k+1) m}\right)}{d_{b j m}},
$$

$$
G_{a j m}=\frac{\left(a_{k j}-a_{\varrho k j}\right)-\left(a_{(k+1) m}-a_{\varrho(k+1) m}\right)}{d_{a j m}},
$$

where $d_{b j m}$ is distance between points corresponding to $b_{k j}$ and $b_{(k+1) m}, d_{a j m}$ is distance between points corresponding to $a_{k j}$ and $a_{(k+1) m}$ and $b_{\varrho k j}, b_{\varrho(k+1) m}, a_{\varrho k j}$, $a_{\varrho(k+1) m}$ are values of these components in equilibrium vacuum medium related to points of approximation.

We see that $G_{b j m}$ and $G_{a j m}$ represent a kind of gradients of deviation from equilibrium of components between various points of approximation. By means of such gradients we should be able to determine a flux $\boldsymbol{J}_{b}$ of $b$ towards $a$. However, such a flux depends also on various intercomponent forces as it was considered in [26]. We do not discuss this in detail. We tend to express our ideas only indicating quantities which can be important in this description. We introduce at this moment a functional

$$
\Psi_{\boldsymbol{n}}=\Psi_{\boldsymbol{n}}\left(\left\{G_{b j m}\right\},\left\{G_{a j m}\right\}, \boldsymbol{J}_{b}\right),
$$

which characterize tendency to generation of new flux of $b$ in given direction determined by the vector $\boldsymbol{n}$, where $\boldsymbol{J}_{b}$ is currently continued flux. Then, generation of new flux is determined by a kind of critical conditions expressed by means of appropriately constructed to this end functional (56) as follows:

$$
\Psi_{\boldsymbol{n}}>\Psi_{\boldsymbol{n} C}
$$

where $\Psi_{\boldsymbol{n} C}$ takes a given value.

In general we should expect that flux in direction $\boldsymbol{n}$ will increase with growth of corresponding absolute values of gradients expressed by $G_{b}, G_{a}$ and should decrease if in another direction $\boldsymbol{n}^{\prime}$ a flux $\boldsymbol{J}_{b} \boldsymbol{n}^{\prime}$ increases. The last property follows from fact that elements of the same component $b$ attract themselves $[24,26]$. As a result of this one flux can predominate another one.

In a stationary chain state we have to do with constant flux $\boldsymbol{J}_{b n}, \boldsymbol{n} \in N_{C H}$ for a group of similar directions. We can consider several cases when the chain state undergoes a change.

Let us consider an open chain state. Then, in front of the chain state considerable excess of $b$ happens which can lead to elongation of the chain state in case when new electron appears before the chain state.

Similar arguments lead to transformation of open chain state to closed chain state since first electron can detect finally presence of the last electron of the chain state within more complex molecular system. Therefore we expect that closed chain states in molecular systems are the most stable. We have discussed previously that closed chain state should accelerate in accordance with Eq. (50). However we expect that the closed chain state can move with an averaged constant velocity within molecular systems. This happens in environment of nuclei and other electrons which can stop acceleration of the chain state.

We admit also the situation when the stationary motion of the chain state can be perturbed by appearing of $A_{E X T}$. Then the functional $\Psi_{\boldsymbol{n}}$ from (56) should be defined by means of $A_{B(k+1)} \cup A_{E X T}$ instead of $A_{B(k+1)}$ only. As a result of this the chain state can slow down or accelerate when energy is provided to it. In particular when the critical condition (57) is violated on directions $\boldsymbol{n}$ which do not belong to previously determined $N_{C H}$ then the chain state can be disrupted. Therefore we expect that closed chain states in molecular systems are the most stable. 
Having introduced potential energies $V$ and $V_{e}$ we assume the following symbolic formula as criterion for evolution of molecular system having a chain state

$$
\left(\frac{\partial}{\partial \boldsymbol{Q}}\right)_{C H}\left(V, V_{e}\right)<0,
$$

where whole formula is symbolic since particular notations need particular interpretations.

The formula (58) means that

$$
\text { or } \begin{aligned}
\left(\frac{\partial}{\partial \boldsymbol{R}}\right)_{C H}(V) \leq 0 \text { and }\left(\frac{\partial}{\partial \boldsymbol{d}}\right)_{C H}\left(V_{e}\right)<0 \\
\quad\left(\frac{\partial}{\partial \boldsymbol{R}}\right)_{C H}(V)<0 \text { and }\left(\frac{\partial}{\partial \boldsymbol{d}}\right)_{C H}\left(V_{e}\right) \leq 0 .
\end{aligned}
$$

Interpretation of the symbol $\left(\frac{\partial}{\partial \boldsymbol{R}}\right)_{C H}$ is associated with corresponding relations. In case of the potential energy $V$ the relation " $<$ " means that $\boldsymbol{R}$ evolves along dynamical path towards averaged lower potential energy. Then, the relation " $\leq$ " means that $\boldsymbol{R}$ can remain in more flat region.

In case of the potential energy $V_{e}$ corresponding relations mean that distances between electrons in the chain state are diminished during evolution or remain constant correspondingly.

We assume in this place that $V_{e}$ is decreasing function of distance between electrons in chain state. However we do not discuss here dynamics of this process. We do it for simplicity. It means that $V_{e}$ represents deeper properties of the chain state in an averaged way. This is done for simplicity in order to express our ideas. Justifications for such an assumption follow from various considerations within vacuum medium mechanics $[26,28]$ and discussion of deeper structure of the vacuum medium [35]. It justifies also fact that relations (59), (60) have symbolic character. They should provide context for more precise formulation of criteria for molecular evolution when theoretical description will be more developed.

If we assume that $V_{e}$ is decreasing function of distance between electrons in chain state than the relation (58) states that evolution of an open energetically molecular system goes towards acceleration of the chain state. This is continued until

$$
\left(\frac{\partial}{\partial \boldsymbol{d}}\right)_{C H}\left(V_{e}\right)=0 .
$$

Let us remember that $V_{e}=V_{e}\left(\boldsymbol{d} ; \boldsymbol{r}_{e}, \boldsymbol{R}, \xi\right)$. Consequently, configuration of other variables than $\boldsymbol{d}$ can make impossible further decrease of this function.

Then, in accordance with (60) evolution of nuclei structure has to be continued along the dynamical path until change of structure by a set of chemical reactions and structural transformations allow again to maintain the condition

$$
\left(\frac{\partial}{\partial \boldsymbol{d}}\right)_{C H}\left(V_{e}\right)<0 .
$$

However we admit also the case that the condition (62) cannot be attained even in the case of various reorganisations of the molecular structure. This could happen when we have to do with an excess of energy which can be pro- vided to the chain state in relation to current molecular configuration. Then we expect that the critical condition (57) could be violated and larger transformation of structure of the chain state could happen.

We see possibility providing of energy to the molecular system by electromagnetic radiation which could be represented by an additional force $\boldsymbol{f}_{\text {rad }}$ added to Eq. (41). This force could have also stochastic character. Then energy could be provided to a subsystem of our molecular system and next transferred by chemical reactions. Consequently, we assume that it is also possible that corresponding energy will be provided by chemical reaction with molecules which during chemical reaction interact particularly with chain state of electrons. Corresponding possibility is manifested then by adding $\mathcal{V}_{\text {Dext }}$ in (51).

Let us consider an example. We assume that various amino acids could appear in prebiotic soup leading to production of peptide chains as a result of action of electromagnetic radiation. Peptide chains are linear since amino acids are joined by peptide bonds. What could happen when we will continue providing of energy to such molecular systems by electromagnetic radiation.

We assume that chain states of electrons will appear in peptide chains when we exceed a critical energy in electronic system. Corresponding chain states of electrons should be closed and rather linear going in opposite directions on both sides of carbon-nitrogen core.

Let us note that we have the relation $D=D\left(v_{C H}\right)$ between averaged distance $D$ between sequential electrons and their averaged velocity $v_{C H}$, considered as decreasing function of velocity for stationary chain state. Consequently providing of energy by radiation leads to increase of $v_{C H}$ and by this decrease of $D$. As a result of this corresponding chain of electrons undergoes contraction and whole molecular structure should be squeezed just owing to this contraction. Linear peptide chain cannot be squeezed arbitrarily. Consequently owing to electrostatic interactions within molecular system we should gradually attain the condition (61). Then, further providing of energy with increasing $v_{C H}$ and constant $D$ should lead to increase of $b$ in the set $B_{F k}$ in front of electron. This follows from fact that constant $D$ does not induce increase of flux $\boldsymbol{J}_{b}$ sufficiently towards $A_{B(k+1)}$. Then amount of $b$ in corresponding zone increases and the set $B_{F k}$ undergo extension.

We would say that providing of excess of energy into chain state of electrons leads to internal stress in molecules leading to its deformation together with contraction of the chain state. Thereby, amount of energy contained in the chain state depends on possibility of maintaining of the contraction of the chain states within this molecule.

Hypothesis 6.1: Possibility of increase of energy of the chain state within the same molecular structure without destroying it depends on ability of this molecular system to maintaining of its structure during contraction of the chain state with increasing energy and by this corresponding squeezing of this structure. 
Let us assume that we have to do in prebiotic soup with larger number of highly excited peptide chains which are situated parallel and close one to another. Then there is probability of violation of the critical condition (57) in direction perpendicular to hitherto evolved chain states of electrons. This is so since amount of $b$ in corresponding zones increases and the sets $B_{F k}$ undergo extension. Consequently, we can expect then invasion of the current chain state into neighbouring atoms of carbon and nitrogen is possible owing to new fluxes $\boldsymbol{J}_{b}$. Such a situation could lead to generation of ribose and adenine, thymine, guanine, cythosine and uracil. All of them are composed predominantly of carbon and nitrogen in cyclic form. Thereby we see that it is probable that pumping of energy into proteins could lead to production of RNA as a consequence of loss of ability for absorption of energy by protein system which is manifested by violation of the condition (57).

There is also another aspect which support this point of view. Cyclic structures containing contracting chain state of electrons admits perhaps larger deformation of these structures during squeezing induced by above contraction of chain states. Consequently we can admit providing to these cyclic structures still larger amount of energy than into linear ones. Therefore we suggest that prebiotic evolution of biological systems was continued by transition from protein into RNA and next to DNA. We expect that this could happen by flowing into these structures just the chain state of electrons.

Hypothesis 6.2: Ribose, adenine, thymine, guanine, cythosine, uracil, and finally RNA could appear as a result of invasion of chain states of electrons placed in highly excited energetically, dense structures of proteins between neighbouring proteins within these structures.

The question is what happens when we will provide energy into chain states of electrons within RNA. We can expect that parts of RNA having cyclic structure can maintain contraction of the chain state to larger degree than in linear protein. We can expect for instance that chain state in cyclic structure, ribose for instance, can diminish its radius by reorganization of corresponding electronic structure. Perhaps such a process in adenine for instance could activate joining to it uracil from another place of RNA which leads to partially double RNA structure. All this perhaps could happen in various interactions with proteins.

It seems that in this way we could consider appearing of tRNA, preribosome and initial forms of mRNA even in absence of DNA. Complex of tRNA and appropriate amino acids could be closed by $\mathrm{mRNA}$ in particular places represented by codons which leads to correspondence of these codons with sequence of nucleotides in mRNA.

Further providing of energy to complex protein-tRNAmRNA could lead to separation of mRNA-protein complex. This complex should evolve by further consumption of energy towards extension and energetic excitation of chain states of electrons present there.
This stage could lead to appearing of DNA by modification of uracil to thymine and ribose to deoxyribose. Then, perhaps more efficient complementarity of nucleotides happens which could induce creation of DNA in form of double helice.

Hypothesis 6.3: Origin of genetic code follows from stage where RNA-protein complex is closed by a kind of mRNA along codons present in part of this RNA corresponding to tRNA within this RNA-protein complex.

Let us assume that molecular structure has attained stage where DNA is present. Then, further providing of energy into corresponding chain states leads to its further squeezing within cyclic structures. This in turn, after exceeding some conditions, could lead to reorganisation of electronic structures resulting breaking of hydrogen bonds between complementary nucleotides and replication of DNA. Thereby we see replication as next stage of evolution of corresponding molecular structure.

Hypothesis 6.4: Driven force for biological evolution is associated with pressure generated by attractor determined by chain state of electrons in molecular systems. This pressure follows flow of chain state in larger and larger open energetically molecular system. Evolution is continued then towards generation of structures where chain states of electrons could attain the largest energy.

We consider here chain state as to be inherent property in living systems. Thereby various processes in cell have to be associated with these states. Let us mention role of chaperones for instance. When proteins are produced then they have to be adapted to needs of living cell. In accordance with our discussion this is possible only when such a protein would have appropriate chain states in order to incorporate it in other processes. Consequently we can formulate also the following hypothesis:

Hypothesis 6.5: Chaperones operate in order to ensure that newly produced proteins obtain appropriate chain states by providing to them energy from ATP. This is associated also with shaping of proteins into appropriate configurations which maintain corresponding chain states. This justifies why in vivo number of protein configurations is considerably reduced and is in accordance with concept expressed in (53) and comments before this formula.

Let us note that whole attractor in molecular system is determined by electronic system as well as by conjugations of electronic and nuclei motion. At this stage of considerations we do not comment on these conjugations. The problem seems to be rather complex. We need appropriate description of nuclei wave function. This in turn depends also on development of more precise description of nuclei in high energy physics in context of vacuum medium mechanics. Then, low energy approximations of corresponding wave functions could lead to more precise determination of attractor within whole molecular systems.

Let us also note that this problem is very important for biology since long lasting evolution leads to homeother- 
mality. This in turn has to be consequence just of conjugation electronic and nuclei motion.

\subsection{Remarks on power of attractor generated by chain state of electrons}

We have estimated that accidental nonlinearities in theoretical description should not lead to determination of attractor governing self-organization in molecular biological systems. We should have conviction that corresponding attractor represents a power. Such a conviction follows from persistence of life. This phenomenon is low energy phenomenon. However, existence of life suggests that corresponding processes are placed in the basin of attraction assigned to an attractor. Persistence of life means that this basin of attraction is large. On the other hand, such a large basin of attraction should be generated by attractor associated with processes of considerable power when they are close to the attractor.

Let us estimate properties of the chain state of electrons. Similar properties should have chain state of positrons. We have example of three-positron chain state as proton. Collision of protons with energy $14 \mathrm{TeV}$ per pair of protons does not lead to disintegration of the proton. We interpret three-positron proton as to be in an asymptotic state with respect to its further evolution. Thereby, taking into account strong resistance of proton against disintegration during high energy collision, we state that power of corresponding attractor is giant considering its action as high energy physics phenomenon. Existence of three positrons in proton gives evidence that the chain state is able to overcome electrostatic repulsion. It also confirms that power of corresponding attractor is large.

We can interpret this power as leading to persistent behavior of electronic structure in low energy range associated with biological evolution and maintaining of integrity of biological organisms. We assume that a system of electrons from biological molecules belongs to basin of attraction assigned to an attractor defined for corresponding multielectron system. It is imaginable that this attractor is similar to that one in proton. In this case electrons should attain very small distances between them. However multielectron system would be unstable in such a case. Stability of this multielectron system is attained then with the aid of whole molecular system and decreasing of distances between electrons is stopped just by structure of the molecular system.

Above discussion supports point of view that the chain state of electrons can be responsible for biological evolution.

\subsection{Remarks on relations between theoretical biology and engineering}

We have shown that vacuum medium mechanics provides foundations for development of theoretical biology. It is expressed by fact that hypotheses justified by properties of the chain state of electrons suggest solutions of problems which are not solved yet in whole biology. Let us mention justification for existence of proteins as first stage of evolution towards protocell. Mentioned hypotheses justify also further evolution towards molecules which contain ring molecules as their parts. This property has RNA as well as DNA. Obtained possibilities for description of life and its evolution mean also that concept of theoretical biology discussed above and based on vacuum medium mechanics stabilizes the vacuum medium mechanics as fundamental theory by increase of its status.

Explanation of phenomena related to cell processes by vacuum medium mechanics means also that such a theory could describe nanotechnological processes in biological cell in a future. This in turn means that theories based on vacuum medium mechanics can be applied to molecular nanotechnology similar to that one attained in cell but realized outside of the cell.

In accordance with our discussion on engineering carried out at the beginning of this work and expressed especially by Remark 1.1, we should lead out this nanotechnology beyond the cell. Consequently, similar nanotechnology could be realized with loss of integrity with corresponding biological system. The term "integrity" is not precisely discussed here. We have discussed such aspects of living organisms in [40-42].

The question is how should we do such a nanotechnology. We state that source of precision depends on chain state of electrons in biological molecules. Thereby, in order to realize similar nanotechnology we should be able to control just the chain state in molecular systems beyond the cell.

It is imaginable to construct a molecular system which contains an attractor or its part based on the chain state of electrons. It is imaginable also that we are able to maintain action of this attractor, perhaps with the aid of another molecular system. Then a part of the first molecular system could have possibility of precise synthesis of some designed molecules or could fulfill other designed functions.

In order to obtain such possibilities on theoretical way we should have at our disposal appropriate theory. Consequently, theoretical chemistry of molecules which contain chain state of electrons seems to be very important on way towards theoretical molecular nanotechnology.

Biological systems efficiently transform energy. Imitating of similar processes on the outside of biological cells is interesting task for engineering. In biological systems corresponding processes are associated with providing of energy by electromagnetic waves or ATP or GTP on the inside of cell. It means that excited electronic states are important in this context. This means also that within theoretical chemistry based on vacuum medium mechanics, theoretical electrochemistry and photochemistry will be of particular importance. We come to the following remark:

Remark 6.1: Theoretical electrochemistry and photochemistry developed on basis of vacuum medium mechanics and describing processes associated with chain states of electrons are considered here as of key importance 
for development of theoretical molecular nanotechnology. In particular this theoretical molecular nanotechnology could lead to development of efficient small scale systems for energy production beyond the biological cell.

Consequently, we could construct beyond the biological organism other systems which act on similar way as in this organism. In particular these systems could be engaged in production of energy. However, owing to loss of integrity we obtain new degree of freedom in order to modify corresponding nanotechnology on ways which are not admissible in living organisms.

We come also to the following conclusion:

Remark 6.2: Attractor governing molecular systems characterized by chain states of electrons and conjugation of electronic and nuclei motion is seen as source of precision in biological processes. This source of precision should be also applied in small scale engineering. In this place we see role of whole theoretical physics for precise determination of this attractor. In particular we should have at our disposal precise high energy models of nuclei in order to obtain by approximation corresponding small energy behavior of their wave function. Just this function will be responsible for conjugation of electronic and nuclei motion.

More general conclusion is that we could imitate biological processes. However, it seems that larger achievements should be associated with entirely new application of laws governing corresponding biological processes towards obtaining of a new quality in comparison with natural evolution. By this we see engineering as continuation of biological evolution however in relation to larger system including environment.

\section{Attempts for development of vacuum medium mechanics leading to elaboration of larger context for description of dynamics}

Importance of description of dynamics for small scale engineering suggests necessity of finding ways for improving of current theories especially with respect to this aspect. Vacuum medium mechanics is not finished yet. At the present stage of development it has interesting properties with respect to description of dynamics. However, we have to look for possibilities of extension of description of dynamics as far as possible. We can expect that current description of attractor governing selforganization could be supplemented by description of more fine properties of vacuum medium mechanics and by this could introduce a new quality.

One suggests to consider more deep structure of vacuum medium. This problem is associated with touching reality which is not accessible by current experimental methods. It means that we should elaborate methodology for creation of description for such a reality. Papers $[32,36]$ are devoted just to this end. In these papers we discuss methodology of constructing of physical theories having in mind correctness of applied mathematics and especially aspect of fitting of theory to reality. This methodology has been elaborated during construction of vacuum medium mechanics.
Following along methodology elaborated in papers $[32,36]$ a model of deep structure of vacuum medium is introduced [35]. Let us assume that described above vacuum medium and components distinguished within it are related to scale $S C_{V M}$. Consequently, we introduce additionally model related to scale $S C_{D V M}<S C_{V M}$. In this approach we consider background vacuum medium corresponding to scale $S C_{D V M}$, where magnetic field and gravitational field are considered. Electric field is not present at this scale. Furthermore the two-ring field is distinguished. Various speculations suggests also that gravitational field could be associated with scale $S C_{V M}$ only and is not present in background vacuum medium.

Within background vacuum medium two kinds of magnetic monopoles can be created. Electric field is associated with polarization within monopole-antimonopole system and therefore this field is related to scale $S C_{V M}$ only and is not present in description related to scale $S C_{D V M}$. As a result of this we consider two opposite magnetic monopoles as the most elementary particles which undergo various interactions mediated by magnetic field and the two-ring field. Summarizing, within description of deep structure of vacuum medium related to scale $S C_{D V M}$ we obtain various hypotheses.

Hypothesis 7.1: Magnetic monopoles are the most elementary particles. We consider two kinds of monopoles with opposite sign.

The component $a$ is considered as component of electron. Let us note that experimental investigations do not reveal any internal structure of electron. Thereby we have obtained hypothesis corresponding to deeper structure of electron as follows:

Hypothesis 7.2: Electron is composed of larger number of magnetic monopoles of the same kind considered as dense fluid which can move within electron during its motion and interactions.

Structure of vacuum medium is considered as network of bounded two opposite monopoles within $S C_{D V M}$ model which can move in background vacuum medium. This suggests that fragment of such a structure can also rotate within remaining stable network of pairs of monopoles represented by $\varrho$-component on more averaged level. Then interactions of rotating structure through a discontinuity surface with resting network of pairs of monopoles govern motion of this rotating structure which is interpreted here as neutrino. This leads to the following hypothesis:

Hypothesis 7.3: Neutrino is composed of rotating with respect to resting vacuum medium structure of pairs of opposite monopoles.

Description of deep structure of vacuum medium has low status. However this description is based on some premises which are discerned in observed experimentally properties of corresponding physical objects.

Development of low status description is important. This provides larger context for description of dynamics. In particular, at this moment we are able to interpret dy- 
namics of internal structure of electron. We are able also to interpret wave functions of elementary particles since they are considered as $\{a, b, \varrho\}(\boldsymbol{X})$, where various components interact. Consequently propagation of the wave function is associated with perturbed network of pairs of monopole-antimonopole systems in which an amount of the same type of monopoles representing $a$ and $b$ components propagate. The wave function is the main factor responsible for selforganization, considered as part of the chain state of electrons. This chain state of electrons interacts with nuclei also by wave function of nuclei. The wave function as such interacts with other fields. Let us mention magnetic field for instance. Thereby deeper interpretation of the wave function can be useful for efficient description of electron-nuclei conjugation during motion. As a result of this full description of attractor in whole molecular system would be possible. Consequently, possibility of interpretation of the wave function can lead to considerable improvement of theoretical descriptions especially related to dynamics.

Above discussion is devoted to indicating how further development of theoretical description of physical reality could be continued towards improving of theoretical nanotechnology.

\section{Concluding remarks}

We see that transition of engineering towards smaller scales induces serious challenge. We discuss such a challenge for theoretical description associated especially with nanotechnology.

The first problem is to provide consistent description for all processes in material in order to make possible creation of appropriate design of structures in small scale. One suggests that this description should be realized within general theory called collection of dynamical systems with dimensional reduction which is a general step towards unified mechanics of materials.

Let us note that foundations for theoretical mechanics of materials are done by the rational mechanics where continuum is formalized and various aspects of constitutive equations theories are developed on formal mathematical way [? ]. This formal mathematical approach is appropriate just for providing basis for correct numerical simulations. However further development of the rational mechanics has been rather stopped. Today this theory is transformed towards rather mathematical mechanics. This means, in particular, that theory of constitutive equations is not discussed sufficiently well as reflecting a physical reality related to processes in materials. Instead of this mathematical aspects are dominant.

In order to apply more appropriate methodology of reflecting of physical reality scale of averaging considered during modelling is formalized. This fact is reflected just in collection of dynamical systems as well as in formulation of continuum with finite-dimensional fields. By this step we can see development of unified mechanics of materials considered in this paper as continuation of formal approach initiated by the rational mechanics [6]. This continuation allows us to unify discrete atomic calculations and small scale models with traditional continuum mechanics approach to mechanics of materials. Thereby, by dimensional reduction procedure we extend formal theoretical description towards models better reflecting physical reality related to processes in materials.

Challenge associated with finding of source of precision for nanotechnological processes was more difficult. Estimation that quantum mechanics and even quantum electrodynamics are not able to indicate attractor governing selforganization in molecular processes suggests replacement of quantum theories by entirely new theory. This new theory should describe better dynamics on the most fundamental level. Therefore vacuum medium mechanics is introduced.

Introduction of new fundamental theory is troublesome especially in case when we expect appropriate consequences for large scale processes and consequences which lead to entirely new, previously not described phenomena. In order to develop large program related to theoretical nanotechnology based on new fundamental theory we should have conviction that such a theory is stable with respect to large set of experimental results realized in various environments. Corresponding analysis of stability of theory is discussed in this paper.

At this moment we have to do with appropriate explanation of experimental results following from high energy physics, astrophysical observations as well as appropriate explanation of mechanisms of biological evolution. For the first time we have suggested mechanism of biological evolution based on the most fundamental elementary particle level.

All this suggests that development of theoretical nanotechnology based on vacuum medium mechanics and proposed here way leading to unified mechanics of materials should be continued having interesting perspectives in a future.

\section{References}

[1] Handbook of Theoretical and Computational Nanotechnology, Eds. M. Rieth, W. Schommers, Forschungszentrum Karlsruhe, Germany 2006.

[2] M.W. Hirsch, S. Smale, Differential Equations, Dynamical Systems and Linear Algebra, Academic Press, New York 1974.

[3] H. Haken, Synergetics, Springer Verlag, Berlin 1977.

[4] J. Kaczmarek, Rep. IMP PAN Gdańsk 514, 1473 (2000).

[5] J. Kaczmarek, Interaction and Multiscale Mechanics 3, 1 (2010).

[6] C. Truesdell, A First Course in Rational Mechanics, Baltimore, Maryland 1972.

[7] J. Kaczmarek, TASK Quaterly 6, 253 (2002).

[8] J. Kaczmarek, Bull. Pol. Acad. Sci. 46, 149 (1998).

[9] G.R. Barsch, J.A. Krumhansl, Phys. Rev. Lett. 53, 1069 (1984).

[10] G.R. Barsch, J.A. Krumhans, Met. Trans. 19A, 761 (1988). 
[11] J.K. Liakos, G.A. Saunders, Philos. Mag. A 46, 217 (1982).

[12] J. Kaczmarek, Int. J. Eng. Sci. 32, 369 (1994).

[13] J. Kaczmarek, Int. J. Eng. Sci. 29, 883 (1991).

[14] J. Kaczmarek, Arch. Mech. 45, 135 (1992).

[15] J. Kaczmarek, Arch. Mech. 45, 167 (1993).

[16] J. Kaczmarek, Arch. Mech. 50, 53 (1998).

[17] J. Kaczmarek, Int. J. Plastic. 19, 1585 (2003).

[18] J. Kaczmarek, W. Ostachowicz, Key Eng. Mater. 293-294, 235 (2005).

[19] J. Kaczmarek, Trans. Inst. Fluid-Flow Machin. 108, 5 (2001).

[20] J. Kaczmarek, Acta Mech. 226, 1419 (2015).

[21] A.S. Dawydow, Quantum Mechanics, PWN, Warszawa 1969.

[22] J.D. Bjorken, S.D. Drell, Relativistic Quantum Fields, McGraw-Hill, New York 1965.

[23] C. Itzykson, J.-B. Zuber, Quantum Field Theory, McGraw-Hill, Singapore 1985.

[24] J. Kaczmarek, Phys. Essays 12, 709 (1999).

[25] J. Kaczmarek, Adv. Stud. Theor. Phys. 2, 393 (2008).

[26] J. Kaczmarek, Adv. Stud. Theor. Phys. 3, 13 (2009).

[27] J. Kaczmarek, Adv. Stud. Theor. Phys. 3, 35 (2009).

[28] J. Kaczmarek, Adv. Stud. Theor. Phys. 4, 413 (2010).

[29] J. Kaczmarek, Adv. Stud. Theor. Phys. 5, 63 (2011).
[30] J. Kaczmarek, Adv. Stud. Theor. Phys. 6, 1355 (2012).

[31] J. Kaczmarek, Adv. Stud. Theor. Phys. 7, 1165 (2013).

[32] J. Kaczmarek, J. Pure Appl. Math. Adv. Appl. 15, 23 (2016)

[33] E. van Beveren, G. Rupp, Material Evidence of a $38 \mathrm{MeV}$ Boson, arXiv:1202.1739 [hep-ph].

[34] Kh.U. Abraamyan, A.B. Anisimov, M.I. Baznat, K.K. Gudima, M.A. Nazarenko, S.G. Reznikov, A.S. Sorin, Observation of the E(38)-Boson, arXiv:1208.3829v2 [hep-ex].

[35] J. Kaczmarek, Malays. J. Phys. 36, 1 (2014).

[36] J. Kaczmarek, Far East J. Appl. Math. 75, 101 (2013).

[37] C.E. Cleland, C.F. Chyba, Origins Life Evolut. Biosph. 32, 387 (2002)

[38] J.H. van Hateren, Origins Life Evolut. Biosph. 43, 491 (2013).

[39] B. Korzeniewski, J. Theor. Biol. 209, 275 (2001).

[40] J. Kaczmarek, Far East J. Appl. Math. 76, 79 (2013).

[41] J. Kaczmarek, Far East J. Appl. Math. 77, 77 (2013).

[42] J. Kaczmarek, Computat. Meth. Sci. Technol. 8, 31 (2002).

[43] P.G. Mezey, Potential Energy Hypersurface, Elsevier, Amsterdam 1987.

[44] P.G. Mezey, Theor. Chim. Acta (Berlin) 58, 309 (1981). 